\title{
Reliability analysis and updating of deteriorating systems with subset simulation
}

\author{
Schneider, Ronald; Thöns, Sebastian; Straub, Daniel
}

Published in:

Structural Safety

Link to article, DOI:

10.1016/j.strusafe.2016.09.002

Publication date:

2017

Document Version

Peer reviewed version

Link back to DTU Orbit

Citation (APA):

Schneider, R., Thöns, S., \& Straub, D. (2017). Reliability analysis and updating of deteriorating systems with subset simulation. Structural Safety, 64, 20-36. https://doi.org/10.1016/j.strusafe.2016.09.002

\section{General rights}

Copyright and moral rights for the publications made accessible in the public portal are retained by the authors and/or other copyright owners and it is a condition of accessing publications that users recognise and abide by the legal requirements associated with these rights.

- Users may download and print one copy of any publication from the public portal for the purpose of private study or research.

- You may not further distribute the material or use it for any profit-making activity or commercial gain

- You may freely distribute the URL identifying the publication in the public portal

If you believe that this document breaches copyright please contact us providing details, and we will remove access to the work immediately and investigate your claim. 
3 Reliability analysis and updating of deteriorating systems 4 with subset simulation

5

Ronald Schneider ${ }^{1, *}$, Sebastian Thöns ${ }^{2}$ and Daniel Straub ${ }^{3}$

${ }^{1}$ Bundesanstalt für Materialforschung und -prüfung (BAM), 7. Safety of Structures, Unter den Eichen 87, 12205 Berlin, Germany

${ }^{2}$ Technical University of Denmark (DTU), Department of Civil Engineering, Brovej, 2800 Kongens Lyngby, Denmark

${ }^{3}$ Technische Universität München (TUM), Engineering Risk Analysis Group, Arcisstr. 21, 80290 Munich, Germany

*Corresponding author: Tel.: +49-30-8104 4372 Email: ronald.schneider@bam.de

\section{Abstract}

An efficient approach to reliability analysis of deteriorating structural systems is presented, which considers stochastic dependence among element deterioration. Information on a deteriorating structure obtained through inspection or monitoring is included in the reliability assessment through Bayesian updating of the system deterioration model. The updated system reliability is then obtained through coupling the updated deterioration model with a probabilistic structural model. The underlying high-dimensional structural reliability problems are solved using subset simulation, which is an efficient and robust sampling-based algorithm suitable for such analyses. The approach is demonstrated in two case studies considering a steel frame structure and a Daniels system subjected to high-cycle fatigue.

\section{Keywords}

Structural reliability, deterioration, Bayesian analysis, inspection, monitoring, subset simulation

\section{Introduction}

Engineering structures are generally subjected to deterioration processes such as fatigue and corrosion, and their structural reliability may thus reduce over time. Predictions of the deterioration progress with quantitative models are uncertain due to the simplified representation of the actual deterioration phenomena, the inherent variability of the influencing parameters and limited information on those parameters. These uncertainties must be addressed when modeling 
deterioration of structures (Lin and Yang 1985; Madsen et al. 1986; Melchers 1999a). Inspections and monitoring are effective means of obtaining information on the actual condition of deteriorating structures. This information should be utilized to reduce uncertainties in probabilistic models. A consistent framework for this task is provided by Bayesian analysis, in which prior probabilistic models are updated with inspection and monitoring outcomes (e.g. Tang 1973; Madsen 1987; Enright and Frangopol 1999). This approach facilitates the quantification of the effect of inspection and monitoring results on the structural reliability, and forms the basis for decisions on maintenance actions and future inspection efforts (e.g. Thoft-Christensen and Sørensen 1987; Faber et al. 2000; Moan 2005; Straub and Faber 2005).

Deterioration processes are generally correlated among structural elements within a system due to common influencing factors, such as environmental conditions and material characteristics (e.g. Moan and Song 2000; Vrouwenvelder 2004; Straub and Faber 2005; Stewart and Mullard 2007). This leads to a correlation among deterioration failures of different elements whose effect on the system reliability has to be assessed as a function of structural redundancy (Straub and Der Kiureghian 2011). Correlation among element deterioration is especially relevant when inspection and monitoring outcomes are considered in the reliability assessment (Vrouwenvelder 2004). An observation at one location within a structure contains more indirect information on the deterioration progress at another location if the correlation among element deterioration is high. For these reasons, the reliability of deteriorating structures should be analyzed and updated considering the structure as a whole.

A number of publications propose methods for computing the time-variant reliability of deteriorating structures, including works by Mori and Ellingwood (1993), Li (1995), Ciampoli (1998), Estes and Frangopol (1999), Stewart and Val (1999) and Li et al. (2015). They consider the time-dependent characteristics of both the load and resistance, but do not account for correlation among element deterioration. More recently, a number of researchers have considered modeling and updating the system deterioration state of structures, taking into account the aspect of spatial correlation among element deterioration (Moan and Song 2000; Li et al. 2004; Faber et al. 2006; Straub 2011b; Qin and Faber 2012; Maljaars and Vrouwenvelder 2014). Therein, the effect of inspections and monitoring results on the probability of either reinforcement corrosion in concrete structures or fatigue failures in steel structures is quantified using Bayesian analysis. However, the impact of deterioration on the structural reliability is not included in these works. Such integrated system reliability analyses are proposed in (Lee and Song 2014; Schneider et al. 2015; Luque and Straub 2016). Lee and Song (2014) consider sequential fatigue failures taking into account the effect of stress redistribution within a structural system. They identify critical failure sequences through a branch-and-bound scheme and iteratively compute and update bounds on the system failure probability. Luque and Straub (2016) and Schneider et al. (2015) propose the use of hierarchical Dynamic Bayesian Network (DBN) models for probabilistically representing deterioration in structural systems and for updating deterioration probabilities as well as the system 
reliability with inspection and monitoring results. While they can be powerful, DBN models are rather demanding in the implementation.

To enable an integrated system reliability analysis of inspected and monitored deteriorating structures, which is computationally efficient and simple to implement, we here develop a framework using two coupled sub-models: a probabilistic system deterioration model, which considers stochastic dependence among element deterioration, and a probabilistic structural model for calculating the failure probability of the weakened system. Motivated by the work of Straub and Der Kiureghian (2011), the system deterioration state is assessed at discrete time intervals and is considered constant within each interval. Information on the deteriorating structure obtained through inspection or monitoring is included in the reliability assessment through Bayesian updating of the system deterioration model. The updated system reliability is then obtained through coupling this updated model with a probabilistic structural model. The resulting structural reliability problems are high-dimensional since they include all (correlated) deteriorating elements. To solve these problems, we apply subset simulation, which is a sampling-based algorithm that can robustly and efficiently handle problems involving a large number of random variables. The method is demonstrated in two case studies considering welded steel structures subjected to fatigue deterioration.

\section{System reliability analysis of deteriorating structures}

\subsection{Deterioration modeling}

Deterioration is modeled at the element level at discrete time steps. An element may be a structural member, a welded connection or a segment of a continuous surface (Straub and Der Kiureghian 2011). The state of deterioration of an element $i$ at time $t$ is represented by a random variable or random vector $D_{i, t}$. For example, in the context of reinforcement corrosion in concrete structures, $D_{i, t}$ may represent the loss of reinforcement cross section. Deterioration of all elements is influenced by a set random variables $\mathbf{X}=\left(X_{1}, \ldots, X_{n}\right)$. The relationship between $\mathbf{X}$ and $D_{i, t}$ is described by a parametric deterioration model $h_{i}$, which is written in generic form as:

$$
D_{i, t}=h_{i}(\mathbf{X}, t)
$$

The joint probability density function (PDF) of $\mathbf{X}$ is denoted by $f_{\mathbf{X}}(\mathbf{x})$. Model uncertainties arising from a simplified representation of the actual deterioration phenomenon are included through additional random variables in $\mathbf{X}$.

All random variables describing the deterioration state of the individual elements at time $t$ are summarized in a vector $\mathbf{D}_{t}=\left(D_{1, t}, \ldots, D_{n_{E}, t}\right)$, where $n_{E}$ is the number of elements considered in the system reliability analysis. This vector represents the overall deterioration state of the structural 
system at time $t$. The relationship between the system deterioration state $\mathbf{D}_{t}$ and the deterioration model parameter $\mathbf{X}$ is described by a function $\mathbf{h}$ as:

$$
\mathbf{D}_{t}=\mathbf{h}(\mathbf{X}, t)=\left(h_{1}(\mathbf{X}, t), \ldots, h_{n_{E}}(\mathbf{X}, t)\right)
$$

\subsection{Modeling dependence among deterioration model parameters}

107 Deterioration of different elements of a structural system is generally interdependent due to the spatial correlation among the uncertain parameters $\mathbf{X}$ influencing their condition. Such spatial dependencies are often due to geometrical proximity, but they mainly exist due to common factors influencing the element condition such as environmental conditions and material characteristics (Luque and Straub 2016). The aspect of spatial correlation of deterioration is especially relevant when inspection and monitoring outcomes are considered in the reliability assessment of deteriorating structures. The effect of such observations on the reliability strongly depends on the spatial correlation among the parameters $\mathbf{X}$. An observation at one location contains more indirect information on the deterioration progress at another location if the correlation among the parameters $\mathbf{X}$ is high.

There is only limited information available on modeling statistical dependence of deterioration in structural systems (e.g. Vrouwenvelder 2004; Malioka 2009; Luque et al. 2016). For example, Vrouwenvelder (2004) estimated the correlation among uncertain parameters influencing fatigue crack growth in welded connections by comparing the scatter of the parameters within one production series to the scatter in the overall population. In most applications, however, correlation among the uncertain parameters $\mathbf{X}$ has to be estimated based at least partially on engineering judgment.

124 Hierarchical models and random field models are commonly applied to represent spatial 125 dependence among the uncertain parameters $\mathbf{X}$. The latter are suitable for representing parameters 126 with inherent spatial variability (e.g. Hergenröder 1992; Stewart and Mullard 2007; Malioka 2009). The random field approach models a spatially varying parameter $X$ as a random variable $X(z)$ at each location $z$, and describes the correlation structure of the different random variables $X(z)$ in terms of a suitable correlation function. Such random fields are typically discretized to enable their numerical representation (see, for example, Betz et al. 2014a). As a result, a random

131 field of a spatially varying parameter is defined by a discrete set of correlated random variables,

132 which are part of $\mathbf{X}$. The joint distribution of the variables in a random field is commonly 133 represented by the Nataf model, also known as the Gaussian copula (Liu and Der Kiureghian 134 1986).

135 Hierarchical models may be applied if common influencing factors can be modeled explicitly (e.g. 136 Maes and Dann 2007; Luque et al. 2016). Such models represent correlation among random 137 variables by defining different levels. The random variables within one level are linked through 


$$
p_{F}(\mathbf{x}, t)=\operatorname{Pr}\left(F_{t} \mid \mathbf{X}=\mathbf{x}\right)=\operatorname{Pr}\left(F_{t} \mid \mathbf{D}_{t}=\mathbf{h}(\mathbf{x}, t)\right)
$$

170 To include the uncertainty in the deterioration model parameters, the total probability theorem is

common influencing factors, which are modeled as random variables at a higher level in the hierarchy. The random variables at the highest level are often called hyper-parameters (see, for example, Maes and Dann 2007). The additional random variables representing common influencing factors in a hierarchical model are included in $\mathbf{X}$. The probability distributions of the random variables in each level are defined conditional on the random variables at the next higher level in the hierarchy. Such a hierarchical dependence structure among the variables in $\mathbf{X}$ can be implemented through the Rosenblatt transformation (Hohenbichler and Rackwitz 1981).

In many instances common influencing factors can, however, not be modeled explicitly. Instead, statistical dependence among the variables in $\mathbf{X}$ is often represented by correlation coefficients. As an example, statistical dependence of fatigue deterioration among welded connections due to common fabrication quality may be modeled by defining a correlation coefficient between the initial crack sizes at different hotspots (Vrouwenvelder 2004). In this case, the Nataf model can be applied to model the joint distribution of the correlated deterioration model parameters.

Parameters influencing deterioration can also be time variant. Such parameters are ideally modeled by stochastic processes (see, for example, Lin and Yang 1985; Straub and Faber 2007; Altamura and Straub 2014). Similar to a random field, a stochastic process represents a time-varying parameter $X$ as a random variable $X(t)$ at each time $t$, and describes the correlation among the random variables $X(t)$ through a suitable correlation function. Continuous-time stochastic processes are discretized to facilitate their numerical representation. The resulting set of correlated random variables is included in $\mathbf{X}$. The joint distribution of the variables in a stochastic process may be represented by the Nataf model. In case a stochastic process has the Markov property, the Rosenblatt transformation may be applied (see, for example, Altamura and Straub 2014).

\subsection{Prior system failure probability}

The system failure probability is assessed conditional on the system deterioration state $\mathbf{D}_{t}$. In agreement with Straub and Der Kiureghian (2011), the deterioration state of a structure is considered constant over a period $\Delta t$. The value of $\Delta t$ depends on how fast deterioration progresses and on the lifetime of the structure. In most applications, a good choice is $\Delta t=1$ year, which is short compared to the typical lifetime of structural systems. Conservatively, the system deterioration state in the period $[t-\Delta t, t]$ is set equal to the state at time $t, \mathbf{D}_{t}$. The relationship between the system deterioration state $\mathbf{D}_{t}$ and the deterioration model parameters $\mathbf{X}$ is described by Equation (2). Let $F_{t}$ denote the event of system failure in the period $[t-\Delta t, t]$. The probability of this event conditional on a realization of the deterioration model parameters $\mathbf{x}$ is written as: applied. The overall probability of system failure in the reference period $[t-\Delta t, t]$ is: 


$$
\operatorname{Pr}\left(F_{t}\right)=\int_{\mathbf{D}_{\mathbf{X}}} \operatorname{Pr}\left(F_{t} \mid \mathbf{X}=\mathbf{x}\right) f_{\mathbf{X}}(\mathbf{x}) d \mathbf{x}=\int_{\mathbf{D}_{\mathbf{X}}} p_{F}(\mathbf{x}, t) f_{\mathbf{X}}(\mathbf{x}) d \mathbf{x}
$$

172 where $\boldsymbol{D}_{\mathbf{X}}$ denotes the domain of definition of $\mathbf{X}$. The total-probability-form of the structural 173 reliability problem is advantageous when random variables not contained in $\mathbf{X}$ also influence the event of system failure $F_{t}$ (Straub and Der Kiureghian 2010). Such random variables are considered in the computation of $p_{F}(\mathbf{x}, t)$. By defining the problem in this form, the deterioration model is decoupled from the structural model.

The conditional system failure probability $p_{F}(\mathbf{x}, t)=\operatorname{Pr}\left(F_{t} \mid \mathbf{D}_{t}=\mathbf{h}(\mathbf{x}, t)\right)$ is computed by performing system reliability analyses of the damaged structure. To this end, the structural model is defined with element properties according to the system deterioration state $\mathbf{D}_{t}=\mathbf{h}(\mathbf{x}, t)$. Random variables influencing the system reliability which are not contained in $\mathbf{X}$ are typically load and resistance parameters. While resistance parameters, such as material strengths and structural dimensions, are usually modeled as time-invariant random variables, load parameters are mostly stochastic processes. However, it is typically sufficient to represent the load process by its extreme value distribution for the reference period $[t-\Delta t, t]$ (Melchers 1999b; Straub and Der Kiureghian 2011). The computation of $p_{F}(\mathbf{x}, t)$ then reduces to a time-invariant reliability analysis of the weakened system. This approach leads to an accurate solution if the load process is ergodic and the maximum loads in two different time periods are statistically independent of each other. This holds at least approximately for most relevant applications.

189 Equation (4) can be transformed into a component reliability problem following Wen and Chen 190 (1987). To this end, we introduce an auxiliary standard uniform random variable $P$ with PDF $f_{P}(p)=1$ and cumulative distribution function $(\mathrm{CDF}) F_{P}(p)=p$. We now note that the following 192 identity holds:

$$
p_{F}(\mathbf{x}, t)=F_{P}\left(p_{F}(\mathbf{x}, t)\right)=\operatorname{Pr}\left(P \leq p_{F}(\mathbf{x}, t)\right)
$$

193 The right hand side of Equation (5) corresponds to a component reliability problem with limit194 state function:

$$
g_{F}(\mathbf{x}, p, t)=p-p_{F}(\mathbf{x}, t)
$$

195 The limit-state function $g_{F}(\mathbf{x}, p, t)$ describes a domain $\Omega_{F}(t)$ in the augmented outcome space of $196 \mathbf{X}$ and $P$ as $\Omega_{F}(t)=\left\{(\mathbf{x}, p): g_{F}(\mathbf{x}, p, t) \leq 0\right\}$. The conditional probability $p_{F}(\mathbf{x}, t)$ can now be 197 expressed as:

$$
p_{F}(\mathbf{x}, t)=\int_{p \in \Omega_{F}(t)} f_{P}(p) d p=\int_{0}^{1} I\left(g_{F}(\mathbf{x}, p, t) \leq 0\right) f_{P}(p) d p
$$




$$
\begin{aligned}
\operatorname{Pr}\left(F_{t}\right) & =\int_{\boldsymbol{D}_{\mathbf{X}}}\left[\int_{0}^{1} I\left(g_{F}(\mathbf{x}, p, t) \leq 0\right) f_{P}(p) d p\right] f_{\mathbf{X}}(\mathbf{x}) d \mathbf{x} \\
& =\int_{\mathbf{D}_{\mathbf{X}}} \int_{0}^{1} I\left(g_{F}(\mathbf{x}, p, t) \leq 0\right) f_{\mathbf{X}}(\mathbf{x}) f_{P}(p) d \mathbf{x} d p \\
& =\int_{(\mathbf{x}, p) \in \Omega_{F}(t)} f_{\mathbf{X}}(\mathbf{x}) f_{P}(p) d \mathbf{x} d p
\end{aligned}
$$

where $I(\cdot)$ is the indicator function: $I(\cdot)=1$ if the condition $(\cdot)$ is true and $I(\cdot)=0$ otherwise. Inserting Equation (7) into Equation (4) gives:

200

201

202

203

204

205

206

207

208

209

210

211

212

213

214

215

$$
\mathbf{X}=\mathbf{T}^{-1}\left(U_{1}, \ldots, U_{n}\right)
$$

$\mathbf{T}(\cdot)$ is a probability preserving one-to-one mapping from the original outcome space of $\mathbf{X}$ to the standard normal space for which the Rosenblatt transformation (Hohenbichler and Rackwitz 1981) 218 or the Nataf transformation (Liu and Der Kiureghian 1986) can be applied (see also Section 2.2).

219 The limit-state function $g_{F}$ is now transformed to $\mathbf{U}$-space as:

$$
G_{F}(\mathbf{u}, t)=u_{0}-\Phi^{-1}\left(p_{F}\left(\mathbf{T}^{-1}\left(u_{1}, \ldots, u_{n}\right), t\right)\right)
$$


$220 G_{F}$ describes the domain $\Omega_{F}^{U}(t)$ in the transformed space as $\Omega_{F}^{U}(t)=\left\{\mathbf{u}: G_{F}(\mathbf{u}, t) \leq 0\right\}$.

221 Therefore, the system failure probability $\operatorname{Pr}\left(F_{t}\right)$ can be expressed in $\mathbf{U}$-space as:

$$
\operatorname{Pr}\left(F_{t}\right)=\operatorname{Pr}\left(G_{F}(\mathbf{U}, t) \leq 0\right)=\int_{\mathbf{u} \in \Omega_{F}^{U}(t)} \varphi_{n+1}(\mathbf{u}) d \mathbf{u}
$$

222 where $\varphi_{n+1}(\mathbf{u})=\prod_{i=0}^{n} \varphi\left(u_{i}\right)$ is the $(n+1)$-variate standard normal PDF and $\varphi(\cdot)$ is the standard 223 normal PDF.

\section{4}

$$
L_{i}(\mathbf{x}, t) \propto \operatorname{Pr}\left(Z_{i}(t) \mid \mathbf{X}=\mathbf{x}\right)
$$

238 Generally, two types of observations can be distinguished: observations providing equality information and observations providing inequality information (Madsen et al. 1986; Straub 2011a).

240 Observations providing equality information are observations that can be described by an 241 observation event such as $Z_{i}(t)=\left\{y_{i}(t)=q_{i}(\mathbf{X}, t)+E_{i}\right\}$, where $y_{i}(t)$ is a measurement of a 242 continuous quantity predicted by the model $q_{i}(\mathbf{X}, t)$ and $E_{i}$ is an additive measurement error with $243 \operatorname{PDF} f_{E_{i}}\left(\epsilon_{i}\right)$. The following equality holds $Y_{i}(t)-q_{i}(\mathbf{X}, t)=E_{i}$, where $Y_{i}(t)$ is the uncertain measurement outcome. In this special but common case, the likelihood of observing $Y_{i}(t)=y_{i}(t)$ given $\mathbf{X}=\mathbf{X}$ is equal to the probability density of the random measurement error $E_{i}$ taking the value $y_{i}(t)-q_{i}(\mathbf{x}, t)$. The corresponding likelihood function can be written as (Straub and

$$
L_{i}(\mathbf{x}, t)=f_{E_{i}}\left(y_{i}(t)-q_{i}(\mathbf{x}, t)\right)
$$


248 In the general case, the likelihood function of an observation $Z_{i}(t)$ of the equality-type is defined 249 as (Straub and Papaioannou 2015b):

$$
L_{i}(\mathbf{x} . t)=f_{Y_{i}(t) \mid \mathbf{X}}\left(y_{i}(t) \mid \mathbf{x}\right)
$$

250 where $f_{Y_{i}(t) \mid \mathbf{X}}\left(y_{i}(t) \mid \mathbf{x}\right)$ is the conditional PDF of the uncertain measurement outcome $Y_{i}(t)$ given $251 \mathbf{X}=\mathbf{x}$, which is typically defined in terms of the PDF of the associated measurement error $E_{i}$. The 252 likelihood function defined in Equation (15) includes the evaluation of the model predicting the 253 measured quantity as in Equation (14).

254 Observations providing inequality information are observations such as "corrosion progress is 255 larger than a limit" or "no fatigue crack detected". An observation $Z_{i}(t)$ of the inequality type is 256 modeled through a function $q_{i}(\mathbf{X}, t)$ as follows (Madsen et al. 1986):

$$
Z_{i}(t)=\left\{q_{i}(\mathbf{X}, t) \leq 0\right\}
$$

257 A function $q_{i}(\mathbf{X}, t)$ of this type can be interpreted as a limit-state function. The corresponding 258 likelihood function is written as (Straub and Papaioannou 2015b):

$$
L_{i}(\mathbf{x}, t)=\operatorname{Pr}\left(Z_{i}(t) \mid \mathbf{X}=\mathbf{x}\right)=I\left(q_{i}(\mathbf{x}, t) \leq 0\right)
$$

259 The value of such a likelihood function is either 0 or 1.

260 All observations obtained in the period $[0, t]$ are expressed by a combined event $Z_{0: t}$ as follows:

$$
Z_{0: t}=\bigcap_{j=1}^{n_{Z}(t)}\left(\bigcap_{i \in S_{j}} Z_{i}\left(t_{j}\right)\right)
$$

261 where $n_{Z}(t)$ is the number of times at which inspections or measurements are performed in the 262 period $[0, t]$ and $S_{j}$ is an index set containing the indices of all observations at time $t_{j}$. The 263 likelihood function describing the relationship between $Z_{0: t}$ and the uncertain deterioration model 264 parameters $\mathbf{X}$ is defined as:

$$
L(\mathbf{x}, t) \propto \operatorname{Pr}\left(Z_{0: t} \mid \mathbf{X}=\mathbf{x}\right)
$$

265 Under the common assumption that all individual observations are statistically independent given 266 the deterioration model parameters $\mathbf{X}=\mathbf{x}, L(\mathbf{x}, t)$ is computed as:

$$
L(\mathbf{x}, t)=\prod_{j=1}^{n_{Z}(t)}\left(\prod_{i \in S_{j}} L_{i}\left(\mathbf{x}, t_{j}\right)\right)
$$


In the case of statistically dependent observations, the combined likelihood has to be formulated as a function of the joint distribution of all measurement errors. Straub and Papaioannou (2015a) provide further details on how to model observations with likelihood functions.

\subsection{Posterior system failure probability}

271 The goal here is to assess the effect of inspection and monitoring outcomes on the failure 272 probability of deteriorating structural systems. In Bayesian analysis, this is achieved by computing 273 the conditional probability of the failure event $F_{t}$ given the observation event $Z_{0: t}$, which is defined 274 as follows:

$$
\operatorname{Pr}\left(F_{t} \mid Z_{0: t}\right)=\frac{\operatorname{Pr}\left(F_{t} \cap Z_{0: t}\right)}{\operatorname{Pr}\left(Z_{0: t}\right)}
$$

275

276

277

278 279

$$
\operatorname{Pr}\left(Z_{0: t}\right)=\int_{\boldsymbol{D}_{\mathbf{X}}} \operatorname{Pr}\left(Z_{0: t} \mid \mathbf{X}=\mathbf{x}\right) f_{\mathbf{X}}(\mathbf{x}) d \mathbf{x}
$$

280

Following Straub (2011a), the integrals in Equations (22) and (23) are transformed such that they 281 282 can be solved using structural reliability methods. This method follows the same principles as presented in Section 2.3 for the computation of $\operatorname{Pr}\left(F_{t}\right)$.

For the purpose of transforming Equations (23), an auxiliary standard normal random variable $P$ is again introduced. In addition, let $c$ be a positive constant that ensures $0 \leq c L(\mathbf{x}, t) \leq 1$ for all x. In this case, the following relationship holds:

$$
c L(\mathbf{x}, t)=F_{P}(c L(\mathbf{x}, t))=\operatorname{Pr}(P \leq c L(\mathbf{x}, t))
$$

286 The right hand side of Equation (24) corresponds to a component reliability problem with limit287 state function:

$$
g_{Z_{e}}(\mathbf{x}, p, t)=p-c L(\mathbf{x}, t)
$$

288 The limit-state function $g_{Z_{e}}(\mathbf{x}, p, t)$ defines a domain $\Omega_{Z_{e}}(t)$ in the augmented outcome space of $289 \mathbf{X}$ and $P$ as $\Omega_{Z_{e}}(t)=\left\{(\mathbf{x}, p): g_{Z_{e}}(\mathbf{x}, p, t) \leq 0\right\}$. The quantity $\mathrm{c} L(\mathbf{x}, t)$ can be interpreted as the 
290 conditional probability of $P$ taking a value in $\Omega_{Z_{e}}(t)$ given $\mathbf{X}=\mathbf{X}$. It can thus be computed by 291 integrating $f_{P}(p)$ over the failure domain $\Omega_{Z_{e}}(t)$ when $\mathbf{X}$ take a value $\mathbf{x}$ :

$$
c L(\mathbf{x}, t)=\int_{p \in \Omega_{Z_{e}}(t)} f_{P}(p) d p=\int_{0}^{1} I\left(g_{Z_{e}}(\mathbf{x}, p, t) \leq 0\right) f_{P}(p) d p
$$

292 Consequently, the likelihood function $L(\mathbf{x}, t)$ can be written as:

$$
L(\mathbf{x}, t)=\frac{1}{c} \int_{0}^{1} I\left(g_{Z_{e}}(\mathbf{x}, p, t) \leq 0\right) f_{P}(p) d p
$$

293 Let $a$ denote the proportionality constant in the likelihood definition given in Equation (19). It 294 follows that:

$$
\operatorname{Pr}\left(Z_{0: t} \mid \mathbf{X}=\mathbf{x}\right)=a L(\mathbf{x}, t)=\frac{a}{c} \int_{0}^{1} I\left(g_{z_{e}}(\mathbf{x}, p, t) \leq 0\right) f_{P}(p) d p
$$

295 Inserting Equation (28) into Equation (23) gives:

$$
\begin{aligned}
\operatorname{Pr}\left(Z_{0: t}\right) & =\frac{a}{c} \int_{\mathbf{D}_{\mathbf{X}}}\left[\int_{0}^{1} I\left(g_{Z_{e}}(\mathbf{x}, p, t) \leq 0\right) f_{P}(p) d p\right] f_{\mathbf{X}}(\mathbf{x}) d \mathbf{x} \\
& =\frac{a}{c} \int_{(\mathbf{x}, p) \in \Omega_{Z_{e}}(t)} f_{\mathbf{X}}(\mathbf{x}) f_{P}(p) d \mathbf{x} d p
\end{aligned}
$$

296 Similarly, it can be shown that the probability of the joint event $F_{t} \cap Z_{0: t}$ can be written as:

$$
\operatorname{Pr}\left(F_{t} \cap Z_{0: t}\right)=\frac{a}{c} \int_{(\mathbf{x}, p) \in \Omega_{F \cap Z_{e}}(t)} f_{\mathbf{X}}(\mathbf{x}) f_{P}(p) d \mathbf{x} d p
$$

297 where the domain $\Omega_{F \cap Z_{e}}(t)$ is defined in the augmented outcome space of $\mathbf{X}$ and $P$ in terms of the 298 limit-state function:

$$
g_{F \cap Z_{e}}(\mathbf{x}, p, t)=p-p_{F}(\mathbf{x}, t) \cdot c L(\mathbf{x}, t)
$$

as $\Omega_{F \cap Z_{e}}(t)=\left\{(\mathbf{x}, p): g_{F \cap Z_{e}}(\mathbf{x}, p, t) \leq 0\right\}$.

300 Inserting Equation (29) and Equation (30) into Equation (21) gives:

$$
\operatorname{Pr}\left(F_{t} \mid Z_{0: t}\right)=\frac{\int_{(\mathbf{x} ; p) \in \Omega_{F \cap Z_{e}}(t)} f_{\mathbf{X}}(\mathbf{x}) f_{P}(p) d \mathbf{x} d p}{\int_{(\mathbf{x} ; p) \in \Omega_{Z_{e}}(t)} f_{\mathbf{X}}(\mathbf{x}) f_{P}(p) d \mathbf{x} d p}=\frac{\operatorname{Pr}\left(g_{F \cap Z_{e}}(\mathbf{X}, P, t) \leq 0\right)}{\operatorname{Pr}\left(g_{Z_{e}}(\mathbf{X}, P, t) \leq 0\right)}
$$


Note that the proportionality constant $a$ vanishes. The numerator and the denominator in Equation (32) correspond to component reliability problems, which can be solved using structural reliability methods.

304 The solution given in Equation (32) can be interpreted as follows. The denominator in Equation (32) corresponds to the probability of an inequality observation event $Z_{e, 0: t}=\left\{g_{z_{e}}(\mathbf{X}, P, t) \leq 0\right\}$ and the numerator is equal to the probability of the joint event $F_{t} \cap Z_{e, 0: t}=\left\{g_{F \cap Z_{e}}(\mathbf{X}, P, t) \leq 0\right\}$. In the context of Bayesian updating, the event $Z_{e, 0: t}$ is equivalent to the original observation event $308 Z_{0: t}$ in the sense that:

$$
\operatorname{Pr}\left(F_{t} \mid Z_{0: t}\right)=\operatorname{Pr}\left(F_{t} \mid Z_{e, 0: t}\right)=\frac{\operatorname{Pr}\left(F_{t} \cap Z_{e, 0: t}\right)}{\operatorname{Pr}\left(Z_{e, 0: t}\right)}
$$

309 For applying structural reliability methods, the component reliability problems defined in Equation 310 (32) are also transformed to standard normal space following Section 2.3. The corresponding limit311 state functions $G_{F \cap Z_{e}}$ and $G_{Z_{e}}$ in $\mathbf{U}$-space are:

$$
G_{F \cap Z_{e}}(\mathbf{u}, t)=u_{0}-\Phi^{-1}\left(p_{F}\left(\mathbf{T}^{-1}\left(u_{1}, \ldots, u_{n}\right), t\right) \cdot c L\left(\mathbf{T}^{-1}\left(u_{1}, \ldots, u_{n}\right), t\right)\right)
$$

312 and

$$
G_{Z_{e}}(\mathbf{u}, t)=u_{0}-\Phi^{-1}\left(c L\left(\mathbf{T}^{-1}\left(u_{1}, \ldots, u_{n}\right), t\right)\right)
$$

313 These limit-state functions respectively describe the domains $\Omega_{F \cap z_{e}}^{U}(t)=\left\{\mathbf{u}: G_{F \cap Z_{e}}(\mathbf{u}, t) \leq 0\right\}$ 314 and $\Omega_{Z_{e}}^{U}(t)=\left\{\mathbf{u}: G_{Z_{e}}(\mathbf{u}, t) \leq 0\right\}$ in the transformed space. Consequently, the probabilities $315 \operatorname{Pr}\left(F_{t} \cap Z_{e, 0: t}\right)$ and $\operatorname{Pr}\left(Z_{e, 0: t}\right)$ can be computed as:

$$
\operatorname{Pr}\left(F_{t} \cap Z_{e, 0: t}\right)=\operatorname{Pr}\left(G_{F \cap Z_{e}}(\mathbf{U}, t) \leq 0\right)=\int_{\mathbf{u} \in \Omega_{F \cap Z_{e}}^{U}(t)} \varphi_{n+1}(\mathbf{u}) d \mathbf{u}
$$

316 and

$$
\operatorname{Pr}\left(Z_{e, 0: t}\right)=\operatorname{Pr}\left(G_{Z_{e}}(\mathbf{U}, t) \leq 0\right)=\int_{\mathbf{u} \in \Omega_{Z_{e}}^{U}(t)} \varphi_{n+1}(\mathbf{u}) d \mathbf{u}
$$

317 The computation of the integrals in Equations (36) and (37) requires the selection of the constant 318 c. A discussion on how to select $c$ is provided in (Betz et al. 2014b; Au et al. 2015; Straub and 319 Papaioannou 2015b). In the general case, the optimal choice is $c=1 / \sup (L(\mathbf{x}, t))$ where $\sup (\cdot)$ 320 is the supremum of the expression $(\cdot)$. In some cases, $\sup (L(\mathbf{x}, t))$ can be readily selected. For 321 instance, in the special case of a single measurement with measurement error $E$, the supremum of 322 the likelihood function is $\sup (L(\mathbf{x}, t))=\max \left(f_{E}(\epsilon)\right)$ where $f_{E}(\epsilon)$ is the PDF of $E$. 


\section{Computing system failure probabilities with subset simulation}

324 Subset simulation, originally proposed by Au and Beck (2001), is an adaptive Monte Carlo method particularly suitable for evaluating the high-dimensional reliability problems defined in Equations (12), (36) and (37). The method is robust and computationally efficient, and it can be implemented relatively easily. The algorithm is here implemented following Papaioannou et al. (2015).

First, consider the computation of the prior system failure probability $\operatorname{Pr}\left(F_{t}\right)=\operatorname{Pr}\left(G_{F}(\mathbf{U}, t) \leq 0\right)$. The basic idea of subset simulation is to express the event $F_{t}$ as an intersection of $M$ intermediate events:

$$
F_{t}=E_{1} \cap E_{2} \cap \ldots \cap E_{M}
$$

331

The intermediate events are nested, i.e. $E_{1} \supset E_{2} \supset \cdots \supset E_{M}=F_{t}$. Consequently, the probability of the event $F_{t}$ can be computed by a product of conditional probabilities:

$$
\operatorname{Pr}\left(F_{t}\right)=\operatorname{Pr}\left(E_{1} \cap E_{2} \cap \ldots \cap E_{M}\right)=\prod_{i=1}^{M} \operatorname{Pr}\left(E_{i} \mid E_{i-1}\right)
$$

In this formulation, the event $E_{0}$ is the certain event. The intermediate events are selected such that the conditional probabilities $\operatorname{Pr}\left(E_{i} \mid E_{i-1}\right), i=1, \ldots, M$ are much larger than $\operatorname{Pr}\left(F_{t}\right)$. In this way, the original problem of evaluating the small probability of the rare event $F_{t}$ reduces to computing a sequence of $M$ larger conditional probabilities.

The intermediate events $E_{i}, i=1, \ldots, M$ are defined as $E_{i}=\left\{G_{F}(\mathbf{U}, t) \leq b_{i}\right\}$ where $b_{1}>b_{2}>$ $\cdots>b_{M}=0$. The values of $b_{i}$ are selected adaptively such that the conditional probabilities are equal to a chosen value $p_{0}$. For this purpose, $N$ samples of $\mathbf{U}$ are simulated at each subset level $i$, conditional on the previous intermediate event $E_{i-1}$. For each generated sample, the limit-state function $G_{F}(\mathbf{u}, t)$ is evaluated and $b_{i}$ is set equal to the $p_{0}$-percentile of the $N$ resulting values of $G_{F}(\mathbf{u}, t)$. This procedure is repeated until the $p_{0}$-percentile becomes negative. At this stage, the failure event $E_{M}=F_{t}$ is reached, for which $b_{M}=0$. The samples conditional on the event $E_{0}$ are obtained by crude Monte Carlo sampling. The samples conditional on the events $E_{i}, i=1, \ldots, M-$ 1 are generated by simulating states of Markov chains starting from the samples conditional on $E_{i-1}$, for which $G_{F}(\mathbf{u}, t) \leq b_{i}$. This is achieved by application of Markov Chain Monte Carlo (MCMC) sampling. An estimator $\hat{P}_{S u S}$ of the prior system failure probability $\operatorname{Pr}\left(F_{t}\right)$ can now be written as:

$$
\operatorname{Pr}\left(F_{t}\right) \approx \hat{P}_{S u S}=p_{0}^{M-1} \hat{P}_{M}
$$

$\hat{P}_{M}$ is the estimate of the conditional probability $\operatorname{Pr}\left(E_{M} \mid E_{M-1}\right)$, which is given by the ratio of the number of samples for which $G_{F}(\mathbf{u}, t) \leq 0$ over the number of samples $N$ simulated conditional on $E_{M-1}$. 
Note that the MCMC samples are generally not statistically independent. Their correlation has an effect on the efficiency and accuracy of subset simulation (see, for example, Au and Beck 2001; Schuëller and Pradlwarter 2007; Papaioannou et al. 2015). It is important to adopt an MCMC sampling algorithm that produces samples with low correlation such that the conditional probabilities $\operatorname{Pr}\left(E_{i} \mid E_{i-1}\right)$ can be estimated with a minimum number of samples. We adopt the adaptive MCMC sampling algorithm of Papaioannou et al. (2015).

The value $p_{0}$ of the conditional probabilities and the number of simulated samples $N$ at each subset level can be chosen freely. A value of $p_{0}=0.1$ is a suitable choice. $N$ should be selected large enough to give accurate estimates of $p_{0}$. Note that the total number of required samples for estimating $\operatorname{Pr}\left(F_{t}\right)$ increases linearly with $-\log _{10}\left(\operatorname{Pr}\left(F_{t}\right)\right)$ when using subset simulation instead of with $1 / \operatorname{Pr}\left(F_{t}\right)$ when using crude Monte Carlo simulation (Au and Beck 2001).

The probabilities $\operatorname{Pr}\left(F_{t} \cap Z_{e, 0: t}\right)=\operatorname{Pr}\left(G_{F \cap Z_{e}}(\mathbf{U}, t) \leq 0\right)$ and $\operatorname{Pr}\left(Z_{e, 0: t}\right)=\operatorname{Pr}\left(G_{Z_{e}}(\mathbf{U}, t) \leq 0\right)$ are calculated accordingly. The posterior system failure probability $\operatorname{Pr}\left(F_{t} \mid Z_{0: t}\right)$ is then computed using Equation (21). Alternatively, the conditional probability $\operatorname{Pr}\left(F_{t} \mid Z_{0: t}\right)=\operatorname{Pr}\left(F_{t} \mid Z_{e, 0: t}\right)$ can be estimated directly with a new subset simulation run following the estimation of $\operatorname{Pr}\left(Z_{e, 0: t}\right)$ (see also Schneider et al. 2013; Straub et al. 2016). For this purpose, a set of nested intermediate events $E_{0} \supset E_{1} \supset \cdots \supset E_{M}$ is defined where $E_{0}=Z_{e, 0: t}, E_{i}=\left\{G_{F \cap Z_{e}}(\mathbf{U}, t) \leq b_{i}\right\}, i=1, \ldots, M$ and $b_{1}>$ $b_{2}>\cdots>b_{M}=0$. The conditional probability $\operatorname{Pr}\left(F_{t} \mid Z_{0: t}\right)$ can now be expressed as:

$$
\operatorname{Pr}\left(F_{t} \mid Z_{0: t}\right)=\operatorname{Pr}\left(E_{1} \cap E_{2} \cap \ldots \cap E_{M} \mid E_{0}\right)=\prod_{i=1}^{M} \operatorname{Pr}\left(E_{i} \mid E_{i-1}\right)
$$

370 The first threshold $b_{1}$ defining the intermediate event $E_{1}=\left\{G_{F \cap Z_{e}}(\mathbf{U}, t) \leq b_{1}\right\}$ is determined from 371 the samples conditional on $E_{0}=Z_{e, 0: t}$, which are obtained as a by-product of estimating $\operatorname{Pr}\left(Z_{e, 0: t}\right)$ 372 with subset simulation. The remaining thresholds $b_{i}, i=2, \ldots, M-1$ are determined following 373 the original subset simulation procedure. When applying this approach, the estimator $\hat{P}_{\text {SuS }}$ defined 374 in Equation (40) provides an estimate of the conditional probability $\operatorname{Pr}\left(F_{t} \mid Z_{0: t}\right)$.

\section{Application a: Zayas frame subjected to fatigue deterioration}

376 We consider the two-dimensional welded steel frame shown in Figure 1, which is known as Zayas 377 frame (Zayas et al. 1980). The critical load scenario is an environmental load $L$. In addition, the 378 frame is subjected to fatigue loads throughout its service life of $T=50$ years. The effect of 379 inspections on the fatigue reliability of the elements and on the reliability of the complete structural 380 system is studied. 


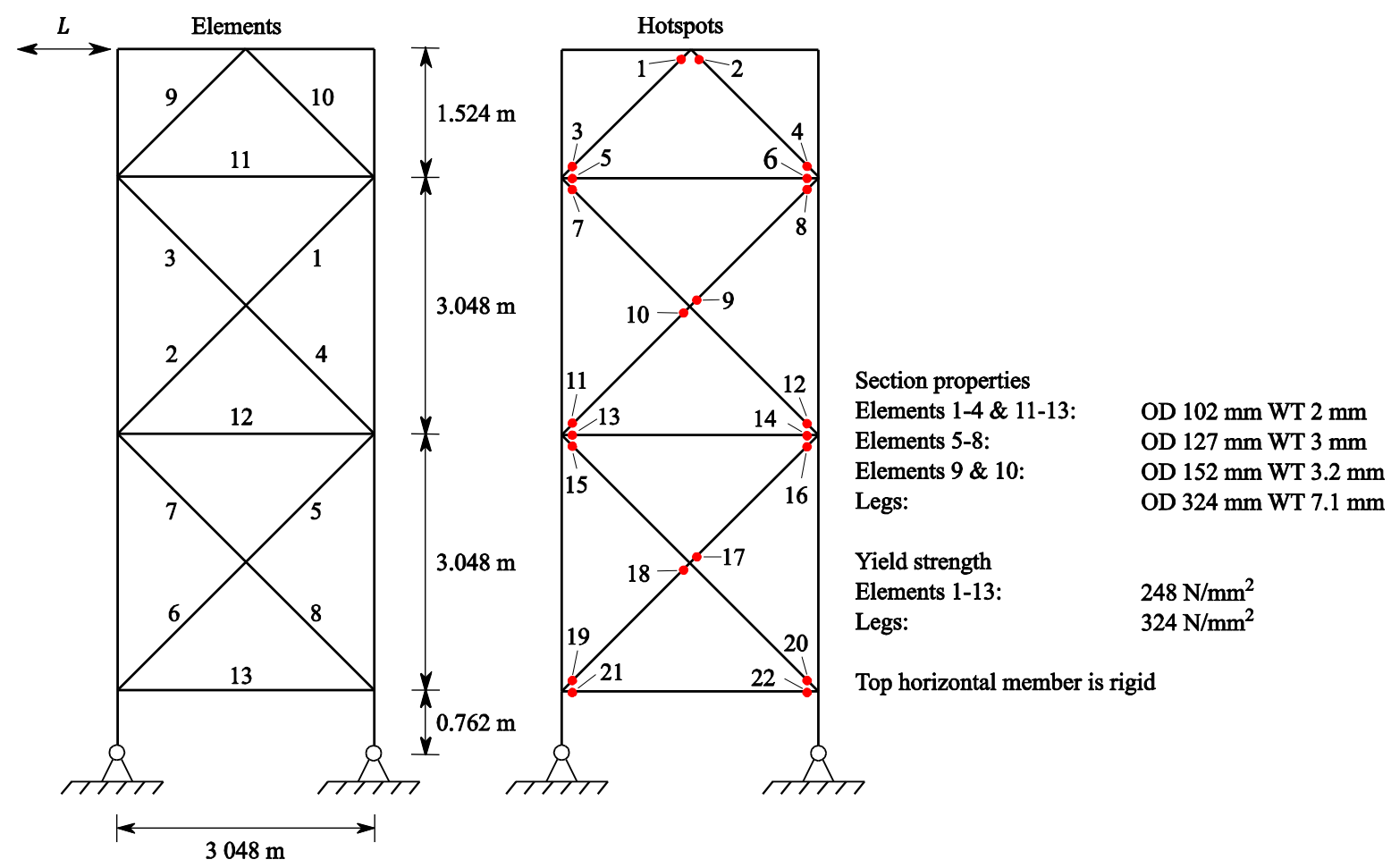

381 Figure 1. Zayas frame (Zayas et al. 1980). OD is the outer diameter and WT is the wall thickness of the

382 tubular steel members.

\section{$383 \quad 5.1 \quad$ System model}

384 The Zayas frame consists of tubular steel elements with welded connections. The state of fatigue deterioration of any element $i$ depends on the condition of the associated welded connections. Fatigue cracks usually develop at locations with local stress concentrations; welded connections are especially vulnerable due to material inhomogeneities, imperfections, high stress concentrations and residual stresses (Fricke 2003). Locations where fatigue cracks may develop are called hotspots. A welded connection may contain multiple hotspots.

390 Fatigue crack growth reduces the capacity of welded connections. In the current example, we assume that fatigue deterioration occurs at the welds connecting the braces with the legs and with

392 the upper horizontal element as well as at the welds at the intersection of the X-braces.

393 Furthermore, we assume that each deteriorating welded connection contains only one critical

394 hotspot. Thus, there are $n_{E}=13$ deteriorating elements and $n_{H}=22$ hotspots as indicated in 395 Figure 1.

396 The approach of Straub and Der Kiureghian (2011) is adopted to determine the reliability of the welded steel structure subjected to fatigue. At system level, no gradual degradation of weld capacities is considered. At a given time $t$, a welded connection has either its full capacity or it has completely lost its capacity because of fatigue crack growth. In the current example, we assume that a welded connection loses its capacity if a fatigue crack at any of the associated hotspots grows beyond a critical size (e.g. Madsen 1997). Thus, the deterioration state of any hotspot $j$ at time $t$ 
402

403

404

405

406

$$
g_{H, j}(\mathbf{x}, t)=a_{c, j}-a_{j}(\mathbf{x}, t)
$$

407

408

409

410

411

412

413

414

415

416

417

Using Equation (2), the system deterioration state $\mathbf{D}_{t}=\left(D_{1, t}, \ldots, D_{n_{E}, t}\right)$ of the Zayas frame can

is modeled by a binary random variable $D_{H, j, t}$, where $\left\{D_{H, j, t}=1\right\}$ is the hotspot fatigue damage event and $\left\{D_{H, j, t}=0\right\}$ is the compliment. The event of fatigue damage of hotspot $j$ at time $t$ is defined by a limit-state function $g_{H, j}(\mathbf{x}, t)$ as $\left\{D_{H, j, t}=1\right\}=\left\{g_{H, j}(\mathbf{X}, t) \leq 0\right\}$ where $\mathbf{X}$ denotes the vector of all uncertain parameters that describe fatigue deterioration of all hotspots considered in the system reliability analysis. $g_{H, j}(\mathbf{x}, t)$ is written as:

where $a_{c, j}$ is the critical crack size and $a_{j}(\mathbf{x}, t)$ is the fatigue crack size at hotspot $j$ at time $t$. $a_{j}(\mathbf{x}, t)$ is computed by means of a probabilistic fatigue crack growth model presented in Section 5.2. $a_{c, j}$ may be defined such that failure modes such as plastic collapse or unstable crack growth are approximately accounted for.

A structural element loses its capacity if any of the associated welded connections loses its capacity. It follows that an element fails as soon as any of the associated hotspots fails due to fatigue deterioration; this corresponds to a series system. The deterioration state of any element $i$ at time $t$ is, therefore, also modeled by a binary random variable $D_{i, t}$ where $\left\{D_{i, t}=1\right\}$ is the event of element fatigue failure and $\left\{D_{i, t}=0\right\}$ is the compliment. From system reliability theory it follows that the event of fatigue failure of element $i$ can be written as:

$$
\left\{D_{i, t}=1\right\}=\bigcup_{j \in C_{i}}\left\{D_{H, j, t}=1\right\}
$$

where $C_{i}$ is an index set containing the indices of all hotspots associated with element $i$. The event of fatigue failure of element $i$ can also be expressed by a limit-state function $g_{i}(\mathbf{x}, t)$ such that $\left\{D_{i, t}=1\right\}=\left\{g_{i}(\mathbf{X}, t) \leq 0\right\} . g_{i}(\mathbf{x}, t)$ is defined as a combination of the individual hotspot limitstate functions $g_{H, j}(\mathbf{x}, t), \forall j \in C_{i}$ as:

$$
g_{i}(\mathbf{x}, t)=\min _{j \in C_{i}} g_{H, j}(\mathbf{x}, t)
$$

The function $h_{i}$ defining the relationship between the fatigue model parameters $\mathbf{X}$ and the element deterioration state $D_{i, t}$ can now be written as:

$$
D_{i, t}=h_{i}(\mathbf{X}, t)=I\left(g_{i}(\mathbf{X}, t) \leq 0\right)
$$
subsequently be calculated as a function of the uncertain fatigue model parameters $\mathbf{X}$. In the current example, $\mathbf{D}_{t}$ is a binary random vector with $2^{n_{E}}$ states.

The Zayas frame is subjected to a time-variant horizontal load whose annual maximum $L$ has the Gumbel distribution with a coefficient of variation (c.o.v.) $\delta_{L}=0.35$. The CDF of $L$ is denoted by 
$F_{L}(l)$. Material and geometry properties are modeled as deterministic parameters as listed in Figure 1. This simplification is reasonable since the uncertainties associated with these quantities are small compared to the uncertainties associated with the system deterioration state and the load $L$. It is thus possible to determine a deterministic ultimate horizontal capacity $r\left(\mathbf{d}_{t}\right)$ of the damaged Zayas frame for any realization of the system deterioration state $\mathbf{D}_{t}=\mathbf{d}_{t}$. Consequently, the conditional probability of system failure $p_{F}(\mathbf{x}, t)$ of the Zayas frame corresponds to the probability that the annual maximum load $L$ exceeds the ultimate capacity $r\left(\mathbf{d}_{t}\right)$ :

$$
\operatorname{Pr}\left(F_{t} \mid \mathbf{D}_{t}=\mathbf{d}_{t}\right)=\operatorname{Pr}\left(r\left(\mathbf{d}_{t}\right) \leq L\right)=1-F_{L}\left(r\left(\mathbf{d}_{t}\right)\right)
$$

The mean of $L$ is selected such that the undamaged Zayas frame has an annual probability of system failure $\operatorname{Pr}\left(F_{t} \mid \mathbf{D}_{t}=\mathbf{0}\right)=1.3 \times 10^{-6}$, leading to $\mu_{L}=62 \mathrm{kN}$.

In the current example, $r\left(\mathbf{d}_{t}\right)$ is computed by performing pushover analysis of the structure with all elements damaged according to $\mathbf{D}_{t}=\mathbf{d}_{t}$, i.e. all elements with fatigue failure are removed from the model used in the pushover analysis. Through such analyses the ultimate capacity of framed steel structures can be quantified. Non-linear effects associated with non-linear material behavior, imperfections, large displacements and deformations (large strains) are modelled explicitly. The analysis captures load redistribution within the structural system resulting from local stiffness changes. It simulates the collapse process of the structural system including initial yielding, formation of plastic hinges, member buckling as well as formation of a global system collapse mechanism (see e.g. Ultiguide 1999; Skallerud and Amdahl 2002).

In the current study, $2^{13}=8192$ pushover analyses are carried out using USFOS (2014) to precalculate the maximum resistance $r\left(\mathbf{d}_{t}\right)$ for all possible realizations of the system deterioration state $\mathbf{D}_{t}$. The corresponding conditional system failure probabilities $\operatorname{Pr}\left(F_{t} \mid \mathbf{D}_{t}=\mathbf{d}_{t}\right)$ are computed according to Equation (46). These failure probabilities have a reference period $\Delta t=1$ year but are independent of time. In the subsequent reliability analysis of the deteriorating Zayas frame, the computation of $p_{F}(\mathbf{x}, t)$ is thus reduced to a lookup operation in which a realization of the fatigue model parameters $\mathbf{x}$ is matched to a pre-calculated conditional system failure probability $\operatorname{Pr}\left(F_{t} \mid \mathbf{D}_{t}=\mathbf{d}_{t}\right)$ at time $t$ by means of Equation (3), i.e. $\mathbf{d}_{t}=\mathbf{h}(\mathbf{x}, t)$.

The influence of individual element failure on the reliability of the Zayas frame depends on the structural importance of the failed element. Following Straub and Der Kiureghian (2011), the structural importance of an element $i$ is quantified in terms of the single-element importance measure $\mathrm{SEI}_{i}$, which is defined as the difference in the failure probability of the undamaged system and the failure probability of the system in which only element $i$ has failed due to fatigue deterioration.

$$
\begin{aligned}
\mathrm{SEI}_{i}= & \operatorname{Pr}\left(F_{t} \mid D_{1, t}=0, \ldots, D_{i-1, t}=0, D_{i, t}=1, D_{i+1, t}=0, \ldots, D_{n_{E}, t}=0\right)- \\
& \operatorname{Pr}\left(F_{t} \mid \mathbf{D}_{t}=\mathbf{0}\right)
\end{aligned}
$$


Table 1 summarizes the single-element importance measures for all deteriorating elements considered in the system reliability analysis of the Zayas frame.

462 Table 1. Single-element importance (SEI) measure and structural importance category of all deteriorating 463 elements of the Zayas frame.

\begin{tabular}{lll}
\hline Element $i$ & SEI $_{i}$ & Structural importance category \\
\hline 1,3 & $1.14 \times 10^{-5}$ & Medium \\
2,4 & $1.06 \times 10^{-5}$ & Medium \\
5,7 & $1.99 \times 10^{-3}$ & High \\
6,8 & $2.00 \times 10^{-3}$ & High \\
9,10 & $7.25 \times 10^{-7}$ & Low \\
11 & $8.26 \times 10^{-8}$ & Low \\
12 & $6.31 \times 10^{-7}$ & Low \\
13 & $2.27 \times 10^{-7}$ & Low \\
\hline
\end{tabular}

464

465 The lower X-braces (elements 5 to 8) are the most important elements followed by the X-braces 466 at the level above (elements 1 to 4). The top braces (elements 9 and 10) and the horizontal braces 467 (elements 11 to 13 ) are the least important elements.

\section{$468 \quad 5.2 \quad$ Fatigue model}

469 In the current example, we adopt the widely used Paris' law (Paris and Erdogan 1963) to describe 470 fatigue crack growth at a given hotspot. For illustration purposes, we consider a through-thickness 471 fatigue crack in an infinite plate subjected to fluctuating stresses in the plane of the plate and 472 orthogonal to the crack. In this case, the fatigue crack is fully characterized by its length $2 a$ and 473 Paris' law is written as:

$$
\frac{d a(n)}{d n}=C(\Delta S(n) \sqrt{\pi a(n)})^{m}
$$

$474 d a(n) / d n$ is the crack growth rate, $n$ is the number of applied fatigue stress cycles, $C$ and $m$ are 475 empirical material parameters and $\Delta S(n)$ is the varying far-field fatigue stress range. The quantity $476 \Delta K=\Delta S(n) \sqrt{\pi a(n)}$ is the stress intensity factor (SIF) range. This model can be extended to 477 account for more complex fatigue crack and hotspot geometries as well as more complex fatigue 478 stress distributions (Straub 2004). If desired, the model can be replaced altogether with a more 479 advanced crack growth model (e.g. Altamura and Straub 2014). This will not affect the method as 480 described in the remainder of the paper.

481 Fatigue loads are generally random and the load sequence $\Delta S(n)$ is ideally modeled by a stochastic 482 process (Altamura and Straub 2014). Under the condition that the fatigue stress process is 


$$
\frac{d a(n)}{d n} \approx E_{\Delta S}\left[C(\Delta S(n) \sqrt{\pi a(n)})^{m}\right]=C(\sqrt{\pi a(n)})^{m} E_{\Delta S}\left[\Delta S(n)^{m}\right]
$$

stationary, ergodic and sufficiently mixing, a simplified approach can be adopted where the crack growth rate $d a(n) / d n$ given by Equations (48) is approximated by its expected value with respect to $\Delta S$ :

486

487

488

489

490

$$
\Delta S_{e}=\left(E_{\Delta S}\left[\Delta S(n)^{m}\right]\right)^{1 / m}=k \Gamma\left(1+\frac{m}{\lambda}\right)^{1 / m}
$$

491

$$
\ln C=-15.84-3.34 m
$$

500 Equation (51) is valid if stresses are given in $\mathrm{N} / \mathrm{mm}^{2}$ and the crack growth rate is given in $\mathrm{m} /$ cycle. 501 In the following, $C$ is modeled as a lognormally distributed random variable. $m$ is thus normal 502 distributed due to the linear relationship between $\ln C$ and $m$.

503 To capture uncertainties in the fabrication quality, the initial crack size $a_{0}$ is modeled as a random 504 variable with exponential distribution. Uncertainties in the calculation of the hotspot stress and in 505 the calculation of the SIF range are captured by introducing lognormal random bias factors $B_{\Delta S}$ 506 and $B_{S I F}$, which are multiplied with the calculated equivalent stress range $\Delta S_{e}$. The one507 dimensional crack growth model given in Equation (48) is rewritten as:

$$
\frac{d a(n)}{d n}=C\left(B_{S I F} B_{\Delta S} \Delta S_{e} \sqrt{\pi a(n)}\right)^{m}
$$


With $a_{j}(n=0)=a_{0, j}$ as initial condition, the differential equation given by Equation (52) is solved for the fatigue crack size $a_{j}$ at hotspot $j$ as a function of time $t$ (Madsen et al. 1986):

$$
a_{j}(\mathbf{X}, t)= \begin{cases}{\left[\left(1-\frac{m_{j}}{2}\right) C_{j} B_{S I F, j}^{m_{j}} B_{\Delta S, j}^{m_{j}} \Delta S_{e, j}^{m_{j}} \pi^{\frac{m_{j}}{2}} v_{j} t+a_{0, j}^{\left(1-\frac{m_{j}}{2}\right)}\right]^{\left(1-\frac{m_{j}}{2}\right)^{-1}},} & m_{j} \neq 2 \\ a_{0, j} \exp \left(C_{j} B_{S I F, j}^{2} B_{\Delta S, j}^{2} \Delta S_{e, j}^{2} \pi v_{j} t\right), & m_{j}=2\end{cases}
$$

510 where $t$ is the time in years, $v_{j}$ is the annual stress cycle rate and $v_{j} t$ is the total number of stress 511 cycles in the period $[0, t] . \Delta S_{e, j}$ is computed as a function of $k_{j}, \lambda_{j}$ and $m_{j}$ according to Equation 512 (50). The vector of all uncertain parameters describing fatigue deterioration of all hotspots 513 considered in the system reliability analysis is defined as:

$$
\mathbf{X}=\left(C_{1}, m_{1}, a_{0,1}, B_{S I F, 1}, B_{\Delta S, 1}, k_{1}, \ldots, C_{n_{H}}, m_{n_{H}}, a_{0, n_{H}}, B_{S I F, n_{H}}, B_{\Delta S, n_{H}}, k_{n_{H}}\right)
$$

514 The same probabilistic models are applied to describe the crack growth model parameters for all 515 hotspots $j=1, \ldots, n_{H}$. They are summarized in Table 2 .

516 Table 2. Probabilistic models of the fatigue crack growth parameters for all hotspots $j=1, \ldots, n_{H}$.

\begin{tabular}{lllll}
\hline Parameter & Dimension & Distribution & Mean & Standard deviation \\
\hline $\ln k_{j}$ & corresponding to $\mathrm{N} / \mathrm{mm}^{2}$ & normal & 2.0 & 0.275 \\
$\lambda_{j}$ & - & deterministic & 0.8 & - \\
$v_{j}$ & $\mathrm{yr}^{-1}$ & deterministic & $5 \cdot 10^{6}$ & - \\
$a_{0, j}$ & $\mathrm{~mm}$ & exponential & 0.11 & 0.11 \\
$a_{c, j}$ & $\mathrm{~mm}$ & deterministic & 20 & - \\
$\ln C_{j}$ & corresponding to $\mathrm{N}$ and $\mathrm{mm}$ & normal & -29.97 & 0.514 \\
$m_{j}$ & - & normal & calculated from $\ln C_{j}=-15.84-3.34 m_{j}$ \\
$B_{\Delta S, j}$ & - & lognormal & 1.0 & 0.1 \\
$B_{S I F, j}$ & - & lognormal & 1.0 & 0.1 \\
\hline
\end{tabular}

518 The mean and standard deviation of the equivalent stress range $\Delta S_{e, j}$ are a function of the 519 distributions of $\ln k_{j}$ and $\ln C_{j}$ through Equations (50) and (51). They are $\mu_{\Delta S_{e, j}}=20.1 \mathrm{~N} / \mathrm{mm}^{2}$ 520 and $\sigma_{\Delta S_{e, j}}=5.65 \mathrm{~N} / \mathrm{mm}^{2}$.

521 Statistical dependence among hotspot fatigue behavior is modeled through correlation coefficients 522 among the fatigue model parameters. In the current example, the fatigue model parameters $a_{0, j}$, $523 C_{j}, k_{j}, B_{\Delta S, j}$ and $B_{S I F, j}$ are equi-correlated among all hotspots $j=1, \ldots, n_{H}$ with correlation 524 coefficients $\rho_{a_{0}}, \rho_{\ln C}, \rho_{\ln k}, \rho_{B_{\Delta S}}$ and $\rho_{B_{S I F}}$. The correlation coefficient $\rho_{a_{0}}$ represents the 525 statistical dependence due to common fabrication quality; $\rho_{\ln C}$ reflects the statistical dependence 
526 due to common material characteristics; $\rho_{\ln k}$ models the statistical dependence due to common 527 loading characteristics; and $\rho_{B_{\Delta S}}$ and $\rho_{B_{S I F}}$ describe statistical dependence due to common 528 uncertainties in the calculation of hotspot fatigue stress ranges and SIF ranges. The joint 529 distribution of all fatigue model parameters in $\mathbf{X}$ is subsequently modeled through a Gaussian 530 copula (Nataf) model (Liu and Der Kiureghian 1986).

531 To study the influence of different levels of statistical dependence among hotspot fatigue behavior, 532 three different dependence cases are considered (low, medium, high), which are defined in terms 533 of the correlation coefficients $\rho_{a_{0}}, \rho_{\ln C}, \rho_{\ln k}, \rho_{B_{\Delta S}}$ and $\rho_{B_{S I F}}$ as listed in Table 3.

534 Table 3. Correlation coefficients among the fatigue crack growth parameters.

\begin{tabular}{llll}
\hline & low dependence & medium dependence & high dependence \\
\hline$\rho_{a_{0}}$ & 0.2 & 0.5 & 0.8 \\
$\rho_{\ln C}$ & 0.2 & 0.5 & 0.8 \\
$\rho_{\text {lnk }}$ & 0.2 & 0.5 & 0.8 \\
$\rho_{B_{\Delta S}}$ & 0.2 & 0.5 & 0.8 \\
$\rho_{B_{S I F}}$ & 0.2 & 0.5 & 0.8 \\
\hline
\end{tabular}

\section{$535 \quad 5.3 \quad$ Inspection model}

536 In the context of fatigue deterioration, relevant inspection outcomes are (a) no detection, (b) 537 detection but no measurement, and (c) detection and measurement of a fatigue crack. These 538 inspection outcomes are directly related to the crack size $a_{j}(\mathbf{X}, t)$ predicted for a given hotspot $j$ 539 at inspection time $t$. In the current study, we consider inspection outcomes of the type (a) and (b). 540 The ability of an inspection method to detect a fatigue crack with a certain size $A=a$ is commonly 541 described by a probability of detection curve $\pi(a)$, which is defined as:

$$
\pi(a)=\operatorname{Pr}(\text { detection of a fatigue crack } \mid A=a)
$$

542 Such a probability of detection curve describes the performance of the applied inspection method; 543 it accounts for uncertain factors such as measurement errors, inspector performance and 544 environmental conditions (Straub 2004). In the current case study, an exponential probability of 545 detection curve is applied:

$$
\pi(a)=1-\exp \left(-a / \lambda_{D}\right)
$$

546 with $\lambda_{D}=1.95 \mathrm{~mm}$. This probability of detection model is representative of magnetic particle 547 inspection (Moan et al. 2000).

548 The likelihood function describing the inspection outcome $Z_{i}(t)=$ ffatigue crack detected at 549 hotpot $j$ at time $t$ \} is thus equal to the probability of detection: 


$$
L_{i}(\mathbf{x}, t)=\pi\left(a_{j}(\mathbf{x}, t)\right)
$$

550 The likelihood function of the complementary inspection outcome $Z_{i}(t)=$ no fatigue crack 551 detected at hotpot $j$ at time $t\}$ is:

$$
L_{i}(\mathbf{x}, t)=1-\pi\left(a_{j}(\mathbf{x}, t)\right)
$$

552 Under the common assumption that individual inspection outcomes are statistically independent

553 given the crack sizes $a_{j}(\mathbf{x}, t), j=1, \ldots, n_{H}$, the combined likelihood function $L(\mathbf{x}, t)$ of all

554 inspection outcomes $Z_{0: t}$ in the time period $[0, t]$ is given by Equation (20). If individual

555 inspections are not statistically independent due to, for example, common influencing factors such

556 as environmental conditions and inspector characteristics, the combined likelihood has to be

557 formulated such that this aspect is captured. Approaches to modeling dependence among

558 inspection outcomes are, for example, presented in (Straub and Faber 2003)(Maljaars and

559 Vrouwenvelder 2014).

560 Since detection/no-detection events provide inequality information, the constant $c$ that ensures $0 \leq$ $561 \quad c L(\mathbf{x}, t) \leq 1$ for all $\mathbf{x}$ can be chosen as $c=1$ (see also Section 3.1).

\section{$562 \quad 5.4 \quad$ Prior system reliability analysis}

563 The prior annual system failure probability $\operatorname{Pr}\left(F_{t}\right)$ of the Zayas frame is computed for each degree 564 of dependence among hotspot fatigue behavior according to Equation (12). The results are shown 565 in Figure 2. The problem is solved using subset simulation as described in Section 4 with 566 conditional probabilities $p_{0}=0.1$ and $N=1000$ samples per subset level. The statistics of the 567 system failure probability are determined from 500 independent simulation runs. This approach is 568 applied in all subsequent analyses presented in this paper. 

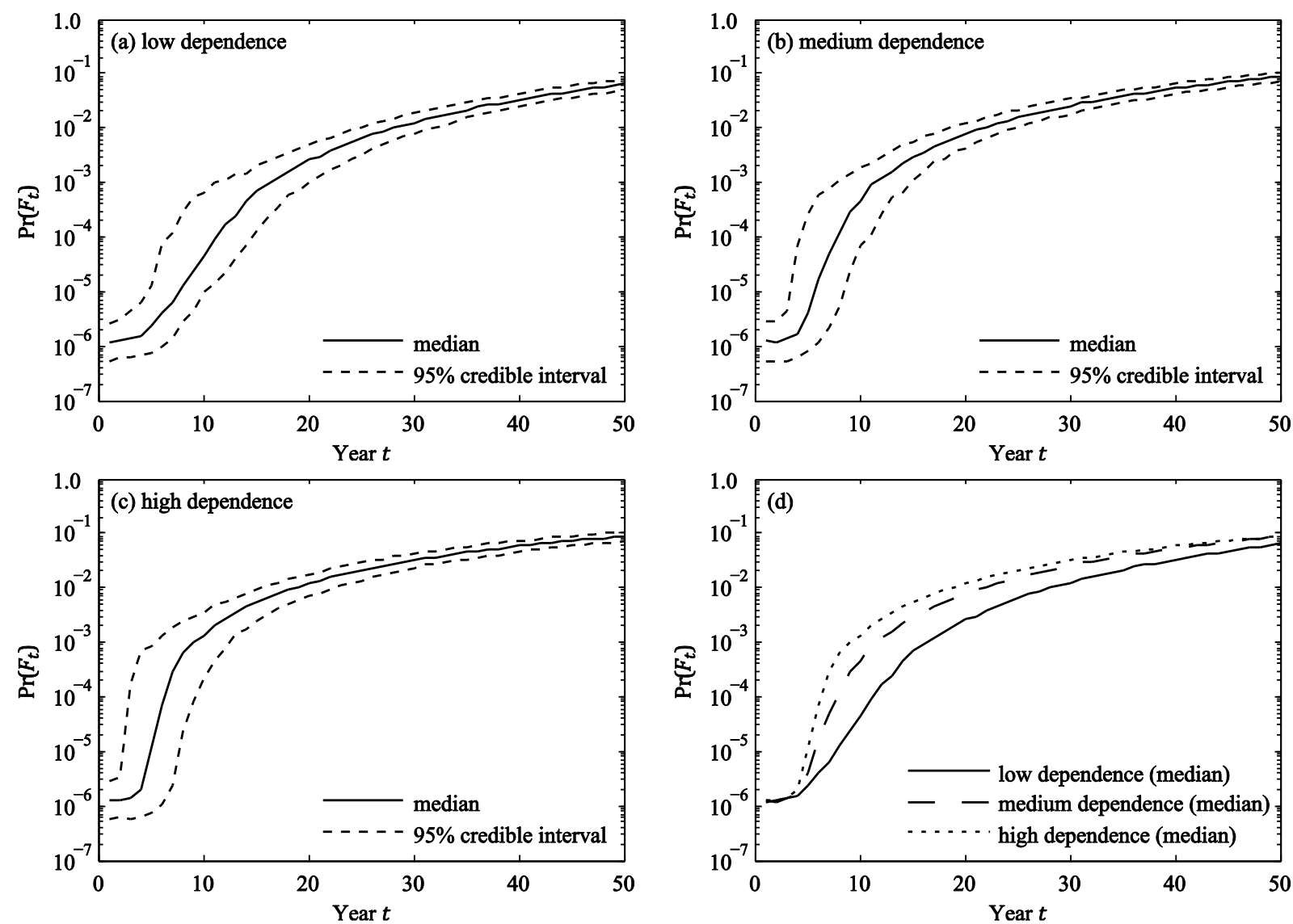

Figure 2. Median and $95 \%$ credible interval of the prior annual system failure probability $\operatorname{Pr}\left(F_{t}\right)$ of the Zayas frame as a function of different degrees of dependence among hotspot fatigue behavior. Computations are performed with subset simulation as summarized in Section 4 with conditional probabilities $p_{0}=0.1$ and $N=1000$ samples per subset level. (d) compares the respective medians of the prior annual system failure probability.

As expected, the annual system failure probability $\operatorname{Pr}\left(F_{t}\right)$ increases with time $t$, due to fatigue deterioration. Furthermore, Figure 2(d) indicates that a higher dependence among hotspot fatigue behavior leads to a larger system failure probability due to an increase in the probability of joint occurrence of several element fatigue failures. This result is expected for a redundant structural system (Straub and Der Kiureghian 2011).

The width of the $95 \%$ credible interval indicates the accuracy of the employed subset simulation.

580 The interval has 0.95 probability of containing the true value of the system failure probability 581 (within the confines of the model). From Figure 2(a) to (c) it can be seen that the accuracy of the 582 computation varies with time $t$ since the number of samples per subset level used in the simulation 583 is the same for all years. Results are less accurate for low values of $t$, because of the associated 584 smaller system failure probability. Note, however, that the variability of the simulated failure 585 probabilities at the beginning of the structure's service life $(t<5 \mathrm{yr})$ is small. In this period, the 586 probability of fatigue failures is very small, and they have little effect on the system failure probability (the failure probability of the undamaged Zayas frame is $\operatorname{Pr}\left(F_{t} \mid \mathbf{D}_{t}=\mathbf{0}\right)=1.3 \cdot 10^{-6}$ ). 


\subsection{Posterior system reliability analysis}

589 In this section, different inspection scenarios in terms of inspection times, coverage and outcomes

590 are considered to study their effect on the reliability of the Zayas frame. Firstly, hotspots

$591\{5,6,13,14,21,22\}$ are inspected at time $t=10$ years. These hotspots are associated with the

592 least important braces of the Zayas frame (see Figure 1 and Table 1). No fatigue cracks are detected

593 during the inspection. The posterior annual system failure probability $\operatorname{Pr}\left(F_{t} \mid Z_{0: t}\right), t=1, \ldots, 50$ are

594 computed for each degree of dependence among hotspot fatigue behavior with subset simulation

595 as described in Section 4 with conditional probabilities $p_{0}=0.1$ and $N=1000$ samples per subset

596 level. The results are shown in Figure 3.
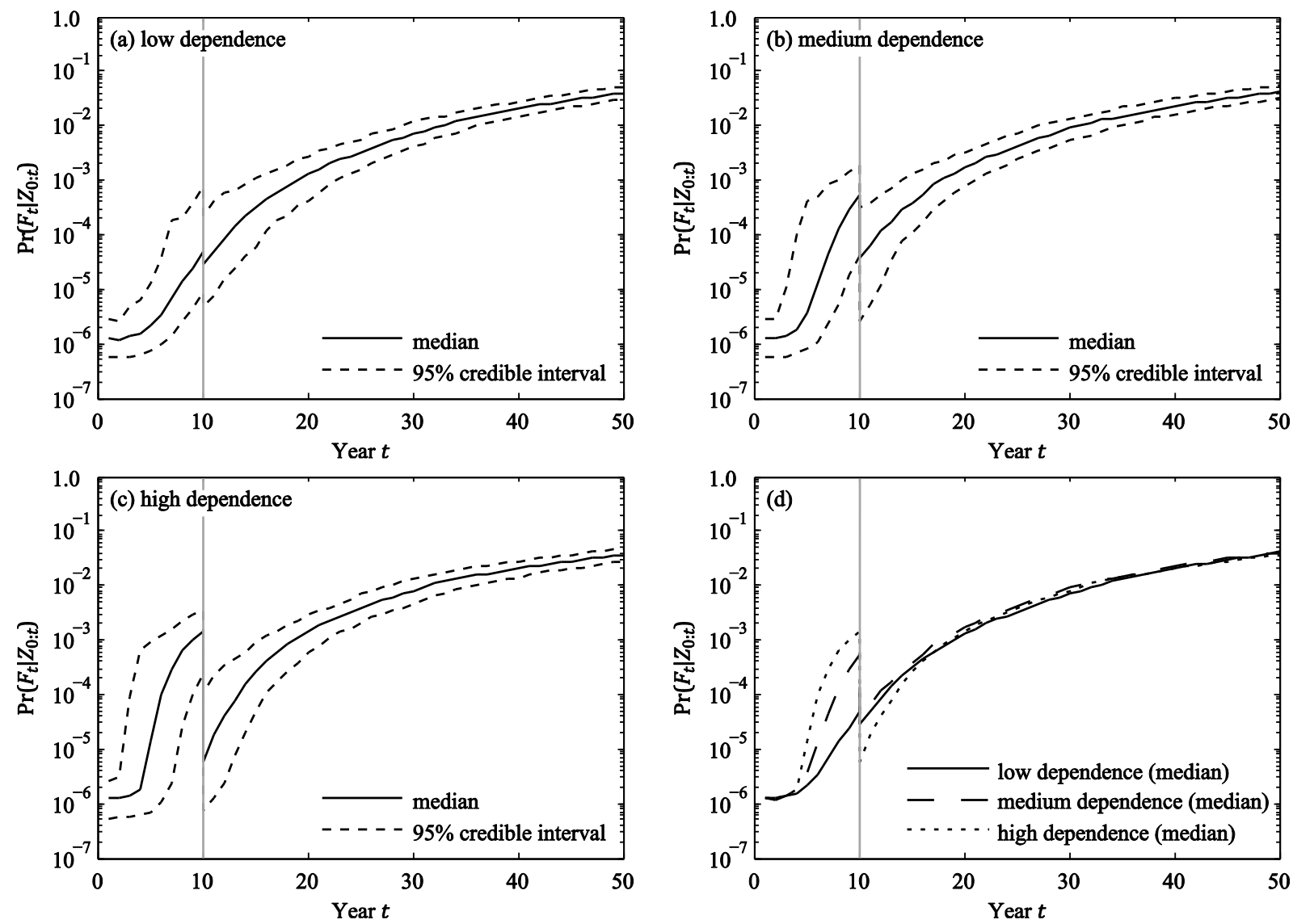

597

598

599

600

601

602

603

604

605

606

Figure 3. Median and $95 \%$ credible interval of the posterior annual system failure probability $\operatorname{Pr}\left(F_{t} \mid Z_{0: t}\right)$ of the Zayas frame as a function of different degrees of dependence among hotspot fatigue behavior. Hotspots $\{5,6,13,14,21,22\}$ are inspected at time $t=10$ years. No fatigue cracks are detected. Computations are performed with subset simulation as summarized in Section 4 with conditional probabilities $p_{0}=0.1$ and $N=1000$ samples per subset level. (d) compares the respective medians of the posterior annual system failure probability.

When considering the posterior medians of the estimated posterior system failure probabilities shown in Figure 3 (a) to (c), it can be seen that the system failure probability reduces after the inspection due to the positive inspection result. The effect increases with increasing degree of dependence among hotspot deterioration behavior. 
Table 4 lists the probabilities $\operatorname{Pr}\left(Z_{e, 0: t}\right)$ and $\operatorname{Pr}\left(F_{t} \mid Z_{0: t}\right)$ computed at time $t=10$ years. The subset 608 simulation $(\mathrm{SuS})$ results are presented together with those from additional Monte Carlo simulations 609 (MCS). The number of model evaluations is also provided for each simulation of $\operatorname{Pr}\left(F_{t} \mid Z_{0: t}\right)$ to 610 indicate the computational efforts, since the accuracy can always be improved by increasing the 611 number of samples. The results in Table 4 show that the probability of the inspection outcome is 612 large. This is because the initial defects at each hotspot considered in the current case study are 613 small, and hence the fatigue cracks are unlikely to grow to a detectable size within the first 10 614 years of the structure's service life.

615 Table 4 Probability of the inspection outcome $\operatorname{Pr}\left(Z_{e, 0: t}\right)$ and the posterior system failure probability $616 \operatorname{Pr}\left(F_{t} \mid Z_{0: t}\right)$ at time $t=10$ years. Subset simulation $(\mathrm{SuS})$ is performed as summarized in Section 4 with 617 conditional probabilities $p_{0}=0.1$ and $N=1000$ samples per subset level. Results in square brackets 618 represent the $95 \%$ credible interval. MCS is performed with $10^{7}$ samples. Results are shown as $95 \%$ 619 confidence interval. The total number of model runs are provided for the computation of $\operatorname{Pr}\left(Z_{e, 0: t}\right)$ and $620 \operatorname{Pr}\left(F_{t} \mid Z_{0: t}\right)$.

\begin{tabular}{lllll}
\hline Case & Method & $\operatorname{Pr}\left(Z_{e, 0: t}\right)$ & $\operatorname{Pr}\left(F_{t} \mid Z_{0: t}\right)$ & \# model runs \\
\hline Low dependence & SuS & {$[0.642 ; 0.7]$} & {$[0.0467 ; 2.3] \cdot 10^{-4}$} & $5.9 \cdot 10^{3}$ \\
& MCS & {$[0.671 ; 0.6716]$} & {$[7.08 ; 8.49] \cdot 10^{-5}$} & $10^{7}$ \\
\hline Medium dependence & SuS & {$[0.659 ; 0.717]$} & {$[0.0267 ; 3.11] \cdot 10^{-4}$} & $5.9 \cdot 10^{3}$ \\
& MCS & {$[0.6875 ; 0.688]$} & {$[0.864 ; 1.01] \cdot 10^{-4}$} & $10^{7}$ \\
\hline High dependence & SuS & {$[0.673 ; 0.734]$} & {$[0.00758 ; 1.08] \cdot 10^{-4}$} & $6.8 \cdot 10^{3}$ \\
& MCS & {$[0.7042 ; 0.7048]$} & {$[2.94 ; 3.79] \cdot 10^{-5}$} & $10^{7}$ \\
\hline
\end{tabular}

622 In the second example, hotspots $\{15,16,17,18,19,20\}$ are inspected at time $t=10$ years. These 623 hotspots are associated with the most important structural members of the Zayas frame (see Figure 6241 and Table 1). We assume again that each inspection results in a no-detection event. The posterior 625 annual system failure probability $\operatorname{Pr}\left(F_{t} \mid Z_{0: t}\right)$ is shown for all three dependence cases in Figure 4. 626 In contrast to the first scenario, an inspection of the most important structural elements has a 627 significant effect on the system reliability regardless of the degree of dependence among element 628 deterioration. 

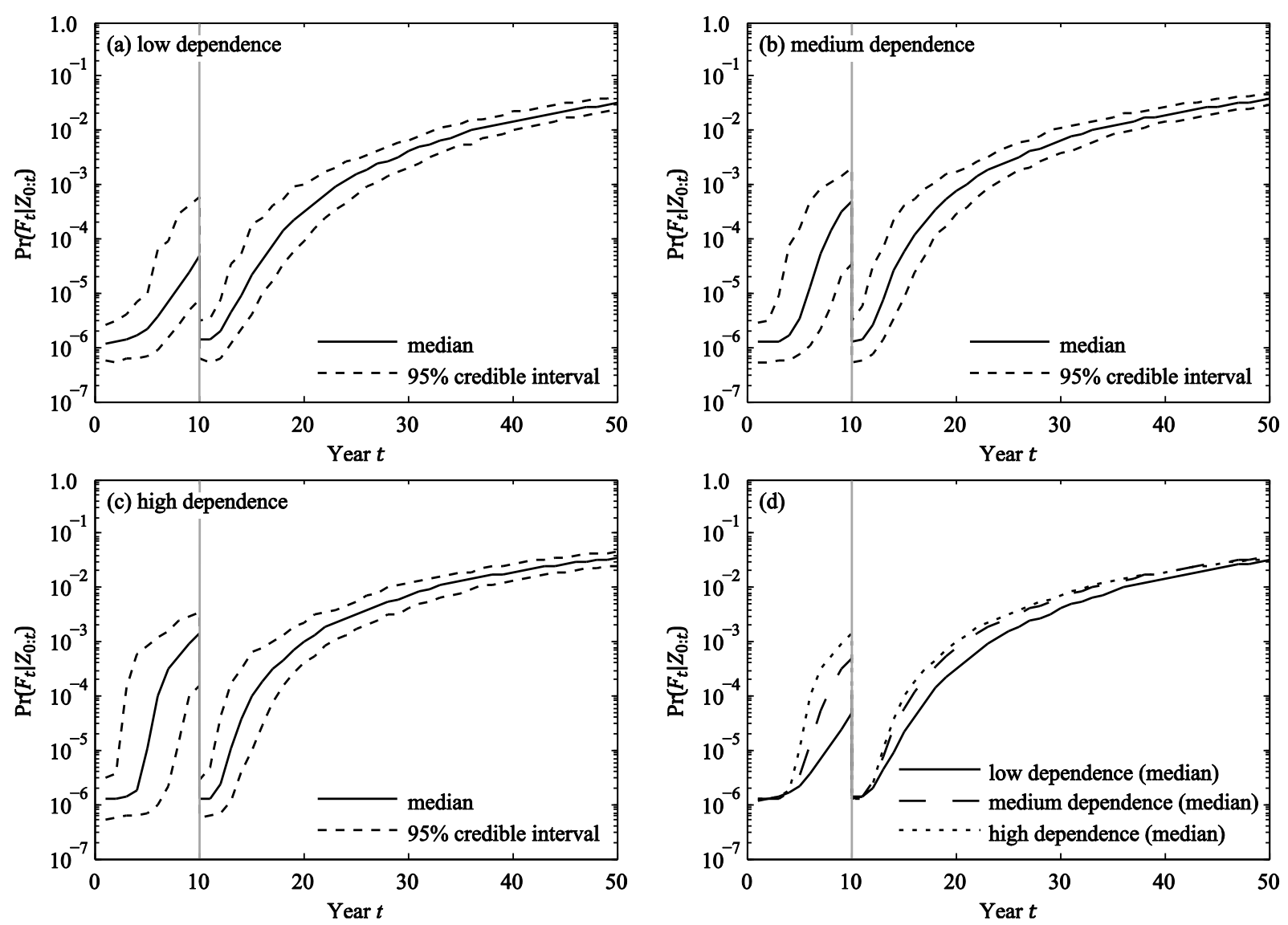

630 Figure 4. Median and $95 \%$ credible interval of the posterior annual system failure probability $\operatorname{Pr}\left(F_{t} \mid Z_{0: t}\right)$ 631 of the Zayas frame as a function of different degrees of dependence among hotspot fatigue behavior. 632 Hotspots $\{15,16,17,18,19,20\}$ are inspected at time $t=10$ years. No fatigue cracks are detected. 633 Computations are performed with subset simulation as summarized in Section 4 with conditional 634 probabilities $p_{0}=0.1$ and $N=1000$ samples per subset level. (d) compares the respective medians of the 635 posterior annual system failure probability.

636 The probability of the inspection outcome $\operatorname{Pr}\left(Z_{e, 0: t}\right)$ and the posterior system failure probability $637 \operatorname{Pr}\left(F_{t} \mid Z_{0: t}\right)$ at year 10 are summarized in Table 5 for each dependence case. The computed 638 probabilities $\operatorname{Pr}\left(Z_{e, 0: t}\right)$ are the same as in the first scenario (see Table 4) because the applied 639 probabilistic models of the crack growth parameters are identical for all hotspots (see Table 2). 640 Comparing the bounds of the SuS and MCS results for $\operatorname{Pr}\left(F_{t} \mid Z_{0: t}\right)$, it is seen that their accuracy is 641 similar even though the number of samples in the SuS is only $N=1000$ samples per subset level. 
Table 5. Probability of the inspection outcome $\operatorname{Pr}\left(Z_{e, 0: t}\right)$ and the posterior system failure probability $643 \operatorname{Pr}\left(F_{t} \mid Z_{0: t}\right)$ at time $t=10$ years. Subset simulation is performed as summarized in Section 4 with 644 conditional probabilities $p_{0}=0.1$ and $N=1000$ samples per subset level. Results represent the $95 \%$ 645 credible interval. MCS is performed with $10^{7}$ samples. Results represent the $95 \%$ confidence interval.

\begin{tabular}{lllll}
\hline Case & Method & $\operatorname{Pr}\left(Z_{e, 0: t}\right)$ & $\operatorname{Pr}\left(F_{t} \mid Z_{0: t}\right)$ & \# model runs \\
\hline Low dependence & SuS & {$[0.642 ; 0.701]$} & {$[0.635 ; 3.15] \cdot 10^{-6}$} & $6.8 \cdot 10^{3}$ \\
& MCS & {$[0.671 ; 0.6716]$} & {$[1.22 ; 3.54] \cdot 10^{-6}$} & $10^{7}$ \\
\hline Medium dependence & SuS & {$[0.656 ; 0.715]$} & {$[0.543 ; 3.12] \cdot 10^{-6}$} & $6.8 \cdot 10^{3}$ \\
& MCS & {$[0.6876 ; 0.6882]$} & {$[1.89 ; 4.53] \cdot 10^{-6}$} & $10^{7}$ \\
\hline High dependence & SuS & {$[0.673 ; 0.73]$} & {$[0.55 ; 2.77] \cdot 10^{-6}$} & $6.8 \cdot 10^{3}$ \\
& MCS & {$[0.7042 ; 0.7048]$} & {$[1.06 ; 3.2] \cdot 10^{-6}$} & $10^{7}$ \\
\hline
\end{tabular}

646

647 In the third scenario, hotspots $\{15,16,17,18,19,20\}$ are again inspected in year 10 . No fatigue 648 cracks are detected at hotspots $\{15,16,17,18\}$ whereas defects are detected at hotspots $\{19,20\}$. 649 The corresponding posterior annual system failure probabilities are shown for all three dependence 650 cases in Figure 5. The system failure probability increases after the inspection since fatigue cracks 651 are detected in welds connecting two of the most important braces with the legs (see Figure 1 and 652 Table 1). The effect is most pronounced in the low dependence case. 

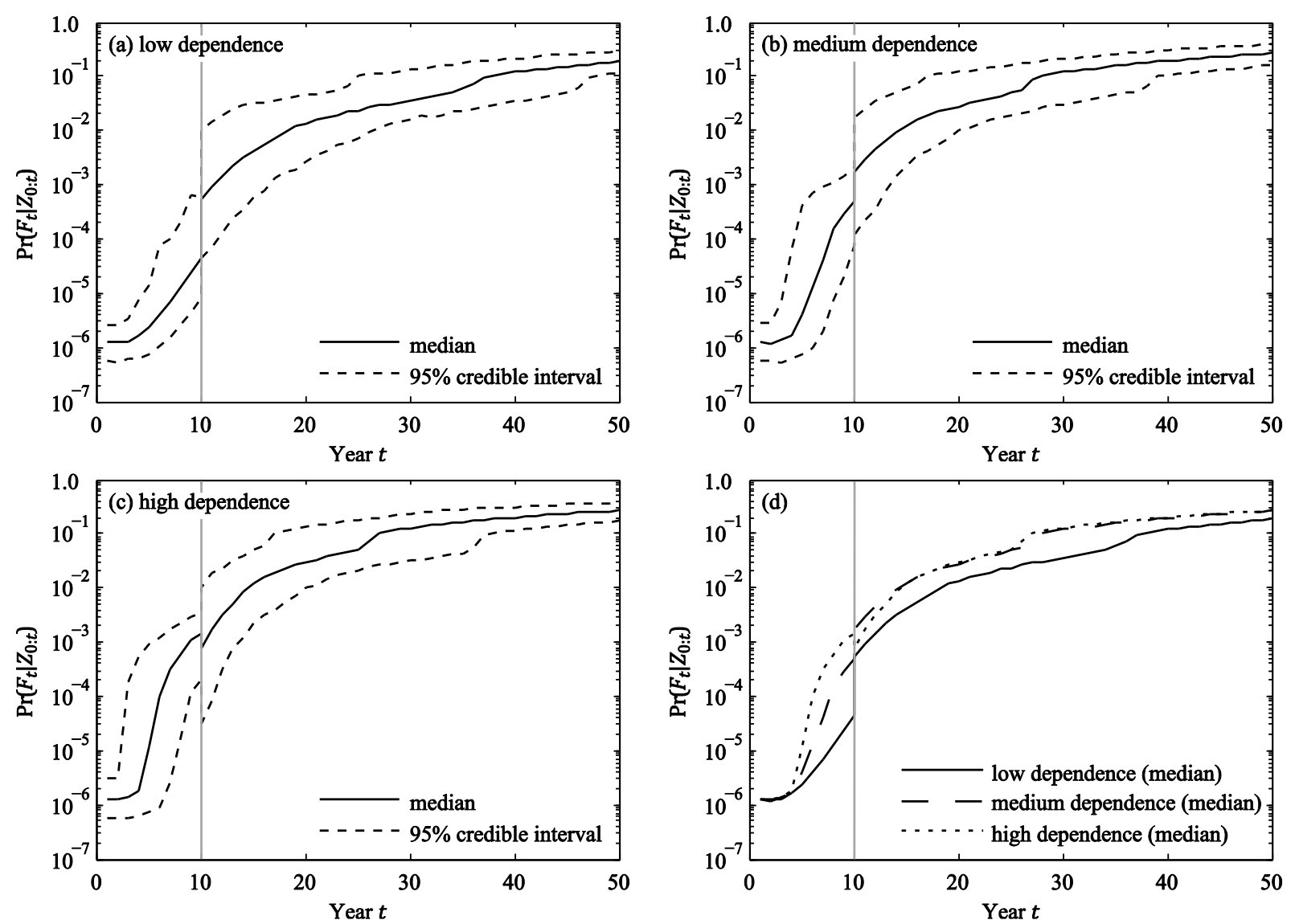

654 Figure 5. Median and $95 \%$ credible interval of the posterior annual system failure probability $\operatorname{Pr}\left(F_{t} \mid Z_{0: t}\right)$ 655 of the Zayas frame as a function of different degrees of dependence among hotspot fatigue behavior. 656 Hotspots $\{15,16,17,18,19,20\}$ are inspected in year 10 . No fatigue cracks are detected at hotspots $657\{15,16,17,18\}$ whereas defects are detected at hotspots $\{19,20\}$. Computations are performed with subset 658 simulation as summarized in Section 4 with conditional probabilities $p_{0}=0.1$ and $N=1000$ samples per 659 subset level. (d) compares the respective medians of the posterior annual system failure probability.

660 Table 6 shows that the current inspection outcome is approximately two orders of magnitude less 661 probable than the no-detection outcomes in the previous scenarios (see Table 4 and Table 5). When 662 comparing the subset simulation results in Table 5 and Table 6 , it can also be seen that the number 663 of model evaluations are similar in both examples although the posterior system failure 664 probabilities $\operatorname{Pr}\left(F_{t} \mid Z_{0: t}\right)$ are multiple orders of magnitude larger in the current example. The 665 reason is that the simulations of the smaller probabilities of the observation event $\operatorname{Pr}\left(Z_{e, 0: t}\right)$ require 666 here more model evaluations. 
Table 6. Probability of the inspection outcome $\operatorname{Pr}\left(Z_{e, 0: t}\right)$ and the posterior system failure probability $668 \operatorname{Pr}\left(F_{t} \mid Z_{0: t}\right)$ at time $t=10$ years. Subset simulation is performed as summarized in Section 4 with 669 conditional probabilities $p_{0}=0.1$ and $N=1000$ samples per subset level. Results represent the $95 \%$ 670 credible interval. MCS is performed with $10^{7}$ samples. Results represent the $95 \%$ confidence interval.

\begin{tabular}{lllll}
\hline Case & Method & $\operatorname{Pr}\left(Z_{e, 0: t}\right)$ & $\operatorname{Pr}\left(F_{t} \mid Z_{0: t}\right)$ & \# model runs \\
\hline Low dependence & $\operatorname{SuS}$ & {$[2.45 ; 5.18] \cdot 10^{-3}$} & {$[0.00429 ; 1.02] \cdot 10^{-2}$} & $7.1 \cdot 10^{3}$ \\
& $\operatorname{MCS}$ & {$[3.66 ; 3.74] \cdot 10^{-3}$} & {$[2.64 ; 3.79] \cdot 10^{-3}$} & $10^{7}$ \\
\hline Medium dependence & $\operatorname{SuS}$ & {$[2.66 ; 5.71] \cdot 10^{-3}$} & {$[0.0121 ; 1.67] \cdot 10^{-2}$} & $6.2 \cdot 10^{3}$ \\
& $\mathrm{MCS}$ & {$[3.82 ; 3.9] \cdot 10^{-3}$} & {$[6.14 ; 7.79] \cdot 10^{-3}$} & $10^{7}$ \\
\hline High dependence & $\mathrm{SuS}$ & {$[2.57 ; 5.78] \cdot 10^{-3}$} & {$[0.0319 ; 9.84] \cdot 10^{-3}$} & $7.0 \cdot 10^{3}$ \\
& $\mathrm{MCS}$ & {$[3.86 ; 3.93] \cdot 10^{-3}$} & {$[2.96 ; 4.13] \cdot 10^{-3}$} & $10^{7}$ \\
\hline
\end{tabular}

671

672 In the last scenario, regular inspections are performed at 10 year intervals. Hotspots associated 673 with elements of each importance category are inspected at each inspection apart from the last 674 inspection where only hotspots associated with the upper braces (low importance category) are 675 inspected (see Figure 1 and Table 1). This inspection strategy ensures that each hotspot is inspected 676 at least once throughout the service life of the structure. The inspection strategy is as follows: 677 hotspots $\{15,16,7,8,5,6\}$ are inspected at time $t=10$ years, hotspots $\{17,18,9,10,13,14\}$ are 678 inspected at time $t=20$ years, hotspots $\{19,20,11,12,21,22\}$ are inspected at time $t=30$ years 679 and hotspots $\{1,2,3,4\}$ are inspected at time $t=40$ years. Each inspection results in a no-detection 680 events. The results are shown in Figure 6. As expected, the positive inspection outcome causes a 681 reduction in the annual system failure probability after each inspection. This effect increases with 682 increasing degree of dependence among hotspot fatigue behavior. 

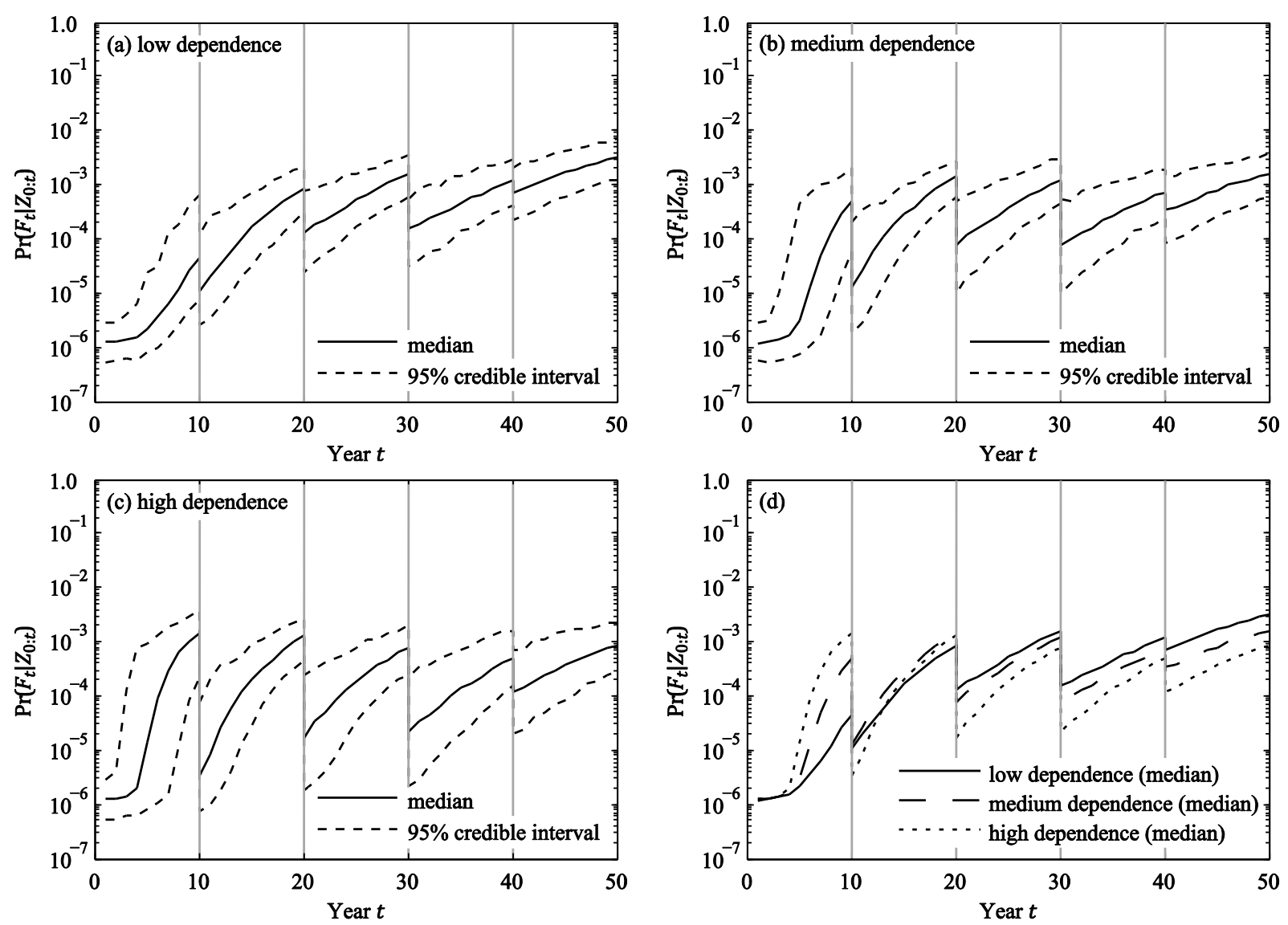

683 Figure 6. Median and $95 \%$ credible interval of the posterior annual system failure probability $\operatorname{Pr}\left(F_{t} \mid Z_{0: t}\right)$ 684 of the Zayas frame as a function of different degrees of dependence among hotspot fatigue behavior. 685 Hotspots $\{15,16,7,8,5,6\}$ are inspected at time $t=10$ years, hotspots $\{17,18,9,10,13,14\}$ are inspected at 686 time $t=20$ years, hotspots $\{19,20,11,12,21,22\}$ are inspected at time $t=30$ years and hotspots $\{1,2,3,4\}$ 687 are inspected at time $t=40$ years. No fatigue cracks are detected. Computations are performed with subset 688 simulation as summarized in Section 4 with conditional probabilities $p_{0}=0.1$ and $N=1000$ samples per subset level. (d) compares the respective medians of the posterior annual system failure probability.

690 Table 7 summarizes the median probability of the inspection outcome $\operatorname{Pr}\left(Z_{e, 0: t}\right)$ after each 691 inspection. Each additional inspection provides more information on the actual condition of the 692 structure. With increasing amount of information, the probability of the inspection outcome $693 \operatorname{Pr}\left(Z_{e, 0: t}\right)$ decreases. It also decreases with decreasing degree of dependence among hotspot 694 fatigue behavior. 
Table 7. Median of the probability of the inspection outcome $\operatorname{Pr}\left(Z_{e, 0: t}\right)$ as a function of the number of 696 inspections and the degree of dependence among hotspot fatigue behavior.

\begin{tabular}{llll}
\hline Year $t$ & low dependence & medium dependence & high dependence \\
\hline 10 & 0.67 & 0.69 & 0.70 \\
20 & 0.42 & 0.47 & 0.52 \\
30 & 0.25 & 0.33 & 0.40 \\
40 & 0.17 & 0.26 & 0.34 \\
\hline
\end{tabular}

\section{Application b: Daniels system subjected to fatigue deterioration}

698 We apply the proposed approach to the idealized structural system shown in Figure 7, known as 699 Daniels system (Daniels 1945). We here assume that the Daniels system consists of welded steel 700 members, which are subjected to fatigue deterioration throughout the structure's service life of $701 T=50$ years. The properties of the Daniels system, in particular the exchangeability of the 702 elements, facilitate numerical investigations.

703

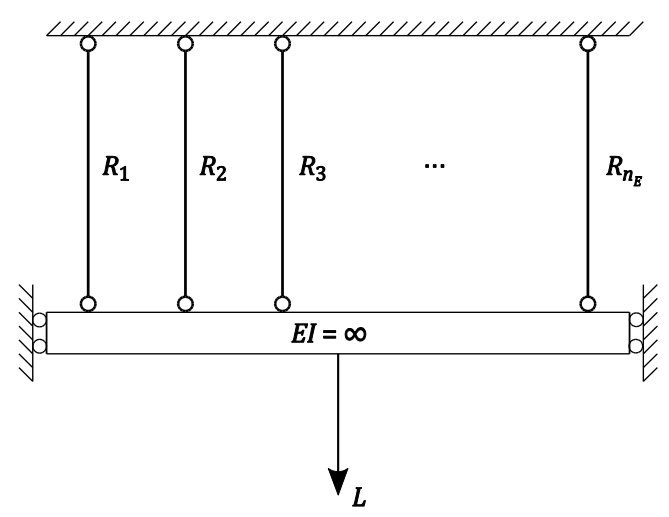

Figure 7. Daniels system with $n_{E}$ elements.

\subsection{System model}

The considered Daniels system consists of $n_{E}=100$ elements with independent and identically distributed (i.i.d.) capacities $R_{i}, i=1, \ldots, n_{E}$. The applied load is shared equally among all elements; its annual maximum is denoted by $L$. In the current example, we assume that each element is associated with one welded connection. Furthermore, we assume that each welded connection contains only one critical hotspot, i.e. $n_{H}=n_{E}=100$.

711 The same deterioration model presented in Section 5 is applied to model fatigue deterioration of

712 the Daniels system. At any time $t$ there are $N_{F, t}$ failed elements and $n_{E}-N_{F, t}$ elements are 713 available to resist the applied loads. Because of the exchangeability of its elements, $N_{F, t}$ represents 714 the deterioration state of the Daniels system at time $t . N_{F, t}$ is computed as a function $h$ of the 715 deterioration model parameters $\mathbf{X}$ as: 


$$
N_{F, t}=h(\mathbf{X}, t)=\sum_{i=1}^{n_{E}} I\left(g_{i}(\mathbf{X}, t) \leq 0\right)
$$

716 where $g_{i}(\mathbf{X}, t)$ is the limit-state function defining the event of fatigue failure of element $i$; see 717 Equation (44). The system failure probability of the Daniels system in the reference period $[t-$ $718 \Delta t, t]$ conditional on a realization of the fatigue model parameters $\mathbf{X}=\mathbf{x}$ can now be written as:

$$
p_{F}(\mathbf{x}, t)=\operatorname{Pr}\left(F_{t} \mid N_{F, t}=h(\mathbf{x}, t)\right)
$$

719 For given probability distributions of the component capacities $R_{i}$ and the annual maximum load $720 L$, the conditional system failure probability $\operatorname{Pr}\left(F_{t} \mid N_{F, t}=k\right), k=0, \ldots, n_{E}$ is readily determined 721 from Daniels system formulation. This failure probability has a reference period $\Delta t=1$ year but 722 it is independent of time $t$. For ductile element behavior (steel elements), the solution is given by 723 (Gollwitzer and Rackwitz 1990):

$$
\operatorname{Pr}\left(F_{t} \mid N_{F, t}=k\right)=\operatorname{Pr}\left(\sum_{i=1}^{n_{E}-k} R_{i} \leq L\right)
$$

724 The right hand side of Equation (61) corresponds to a component reliability problem, which can 725 be solved using structural reliability methods.

726 In the current example, the component capacities $R_{i}, i=1, \ldots, n_{E}$ are modeled as i.i.d. normal 727 random variables with c.o.v. $\delta_{R}=0.15$. The annual maximum of the applied load $L$ is modeled as 728 a lognormal random variable with c.o.v. $\delta_{L}=0.25$. The ratio of the mean values of $n_{E} R_{i}$ and $L$ is 729 selected such that the undamaged Daniels system has a probability of failure $\operatorname{Pr}\left(F_{t} \mid N_{F, t}=0\right)=$ $7301.3 \times 10^{-6}$. The resulting ratio is $n_{E} \mu_{R_{i}} / \mu_{L}=3.09$. $\operatorname{Pr}\left(F_{t} \mid N_{F, t}=k\right), k=0, \ldots, n_{E}$ is pre731 calculated for each realization of the system deterioration state by solving Equation (61) using the 732 first-order reliability method (FORM). The results are illustrated in Figure 8. In the subsequent 733 reliability analysis of the deteriorating Daniels system, the computation of $p_{F}(\mathbf{x}, t)$ is again 734 reduced to a lookup operation. 


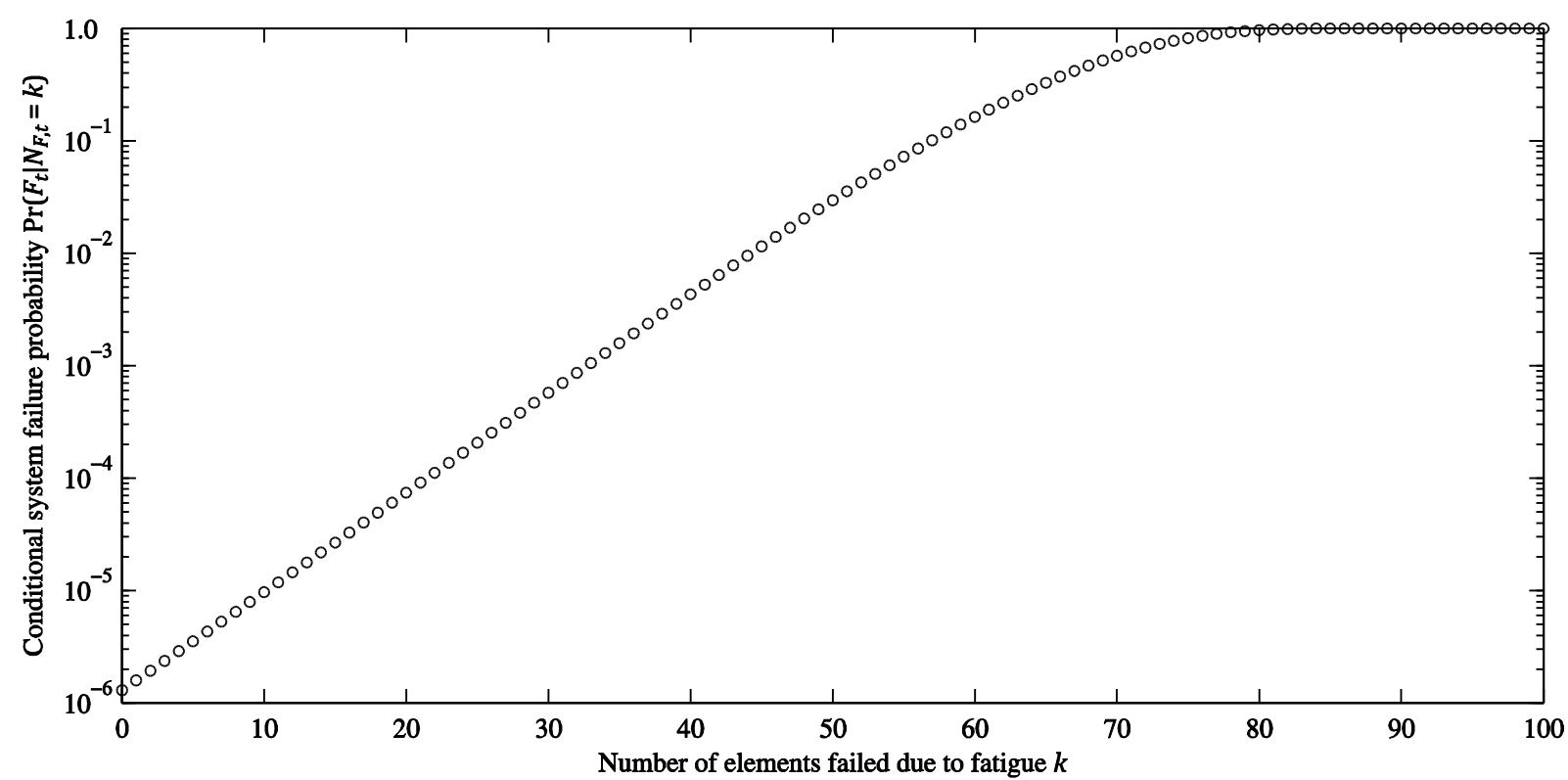

735 Figure 8. Failure probability of the Daniels system as a function of the number of elements failed due to 736 fatigue.

737 Each element of the Daniels system is equally important due to the perfect load sharing among the 738 structural elements. The single element importance measure of an individual element $i$ of the 739 Daniels system is $\mathrm{SEI}_{i}=\operatorname{Pr}\left(F_{t} \mid N_{F, t}=1\right)-\operatorname{Pr}\left(F_{t} \mid N_{F, t}=0\right)=2.9 \times 10^{-7}$. The Daniels system 740 is highly redundant with respect to single element failure when compared to the Zayas frame 741 studied in Section 5 where failure of elements of the highest importance category lead to a 742 significant reduction in system reliability, see Table 1.

\section{$743 \quad 6.2$ Prior system reliability analysis}

744 The computed prior annual system failure probability $\operatorname{Pr}\left(F_{t}\right)$ of the Daniels system is shown in 745 Figure 9 for each degree of dependence among hotspot fatigue. Computations are performed with 746 subset simulation as summarized in Section 4 with conditional probabilities $p_{0}=0.1$ and $N=$ 7471000 samples per subset level. 

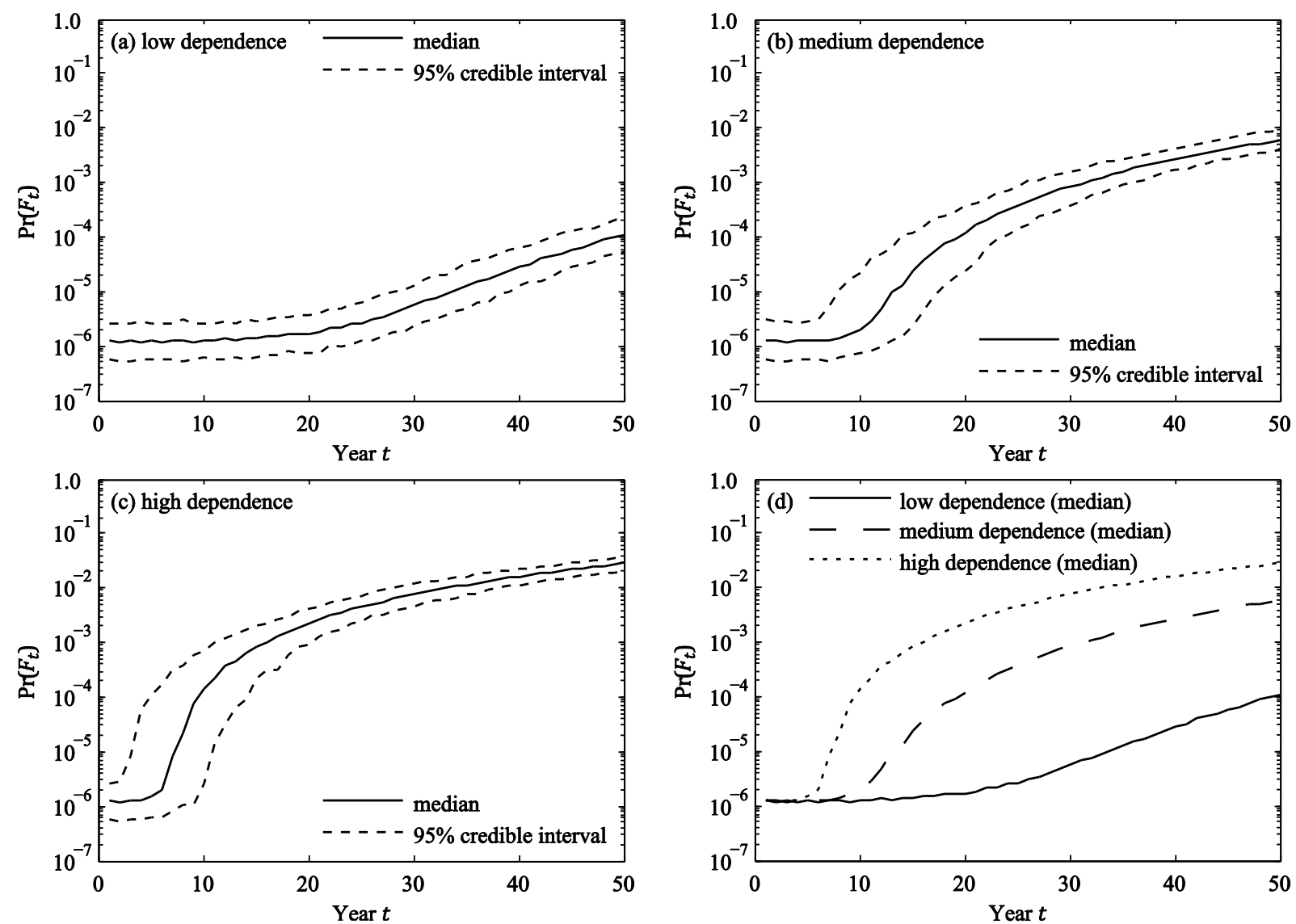

Figure 9. Median and $95 \%$ credible interval of the prior annual system failure probability $\operatorname{Pr}\left(F_{t}\right)$ of the Daniels system as a function of different degrees of dependence among hotspot fatigue behavior. Computations are performed with subset simulation as summarized in Section 4 with conditional probabilities $p_{0}=0.1$ and $N=1000$ samples per subset level. (d) compares the respective medians of the prior annual system failure probability.

In general, a large dependence among element deterioration behavior increases the probability of joint occurrence of more than one element deterioration failures. Figure 9 shows that this behavior has a significant influence on the reliability of the Daniels system. This outcome is expected for a structural system with a large redundancy. In contrast, the results computed for the Zayas frame show that the influence of correlation among element deterioration failures is less pronounced for structural systems with limited or no redundancy (see Figure 2).

\subsection{Posterior system reliability analysis}

760 To study the effect of inspections on the reliability of the Daniels system, different inspection 761 scenarios in terms of inspection times and coverage are considered. Each inspection is assumed to

762 result in a no detection event. The same inspection model as presented in Section 5.3 is applied.

763 In the first scenario, hotspots $\{1$ to 10$\}$ are inspected at time $t=10$ years. The updated annual 764 system failure probabilities $\operatorname{Pr}\left(F_{t} \mid Z_{0: t}\right)$ of the Daniels system are shown in Figure 10 for each 765 degree of dependence among hotspot fatigue behavior. In all three dependence cases, the system 
failure probability decreases after the inspection due to the positive inspection outcome. After the inspection, the system failure probability is reduced to its lower limit, which corresponds to the reliability of the undamaged structure at the beginning of its service live. The subsequent increase in the annual system failure probability is most pronounced in the high-dependence case.
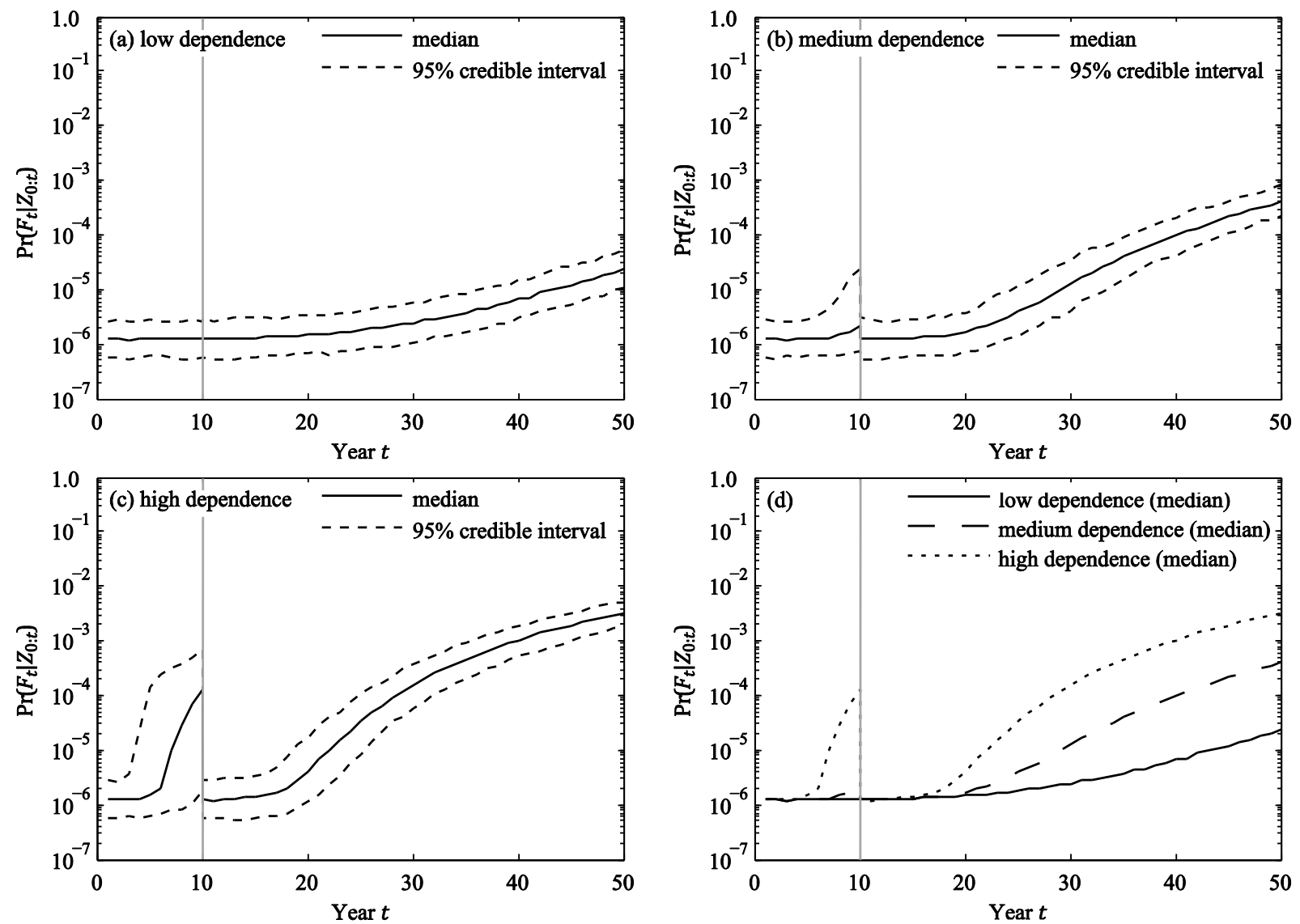

770

771

772

773

774

775

Figure 10. Median and 95\% credible interval of the posterior annual system failure probability $\operatorname{Pr}\left(F_{t} \mid Z_{0: t}\right)$ of the Daniels system as a function of different degrees of dependence among hotspot fatigue behavior. hotspots $\{1$ to 10$\}$ are inspected in year 10 . No fatigue cracks are detected. Computations are performed with subset simulation as summarized in Section 4 with conditional probabilities $p_{0}=0.1$ and $N=1000$ samples per subset level. (d) compares the respective medians of the posterior annual system failure probability.

In the second scenario, different sets of hotspots are inspected at 10 year intervals. The inspection strategy is as follows: hotspots $\{1$ to 10$\}$ are inspected at time $t=10$ years, hotspots $\{11$ to 20$\}$ are inspected at time $t=20$ years, hotspots $\{21$ to 30$\}$ are inspected at time $t=30$ years and hotspots $\{31$ to 40$\}$ are inspected at time $t=40$ years. The results are shown in Figure 11. In all three dependence cases, the posterior annual system failure probability is close the annual failure probability of the undamaged structures after all inspections are performed. 

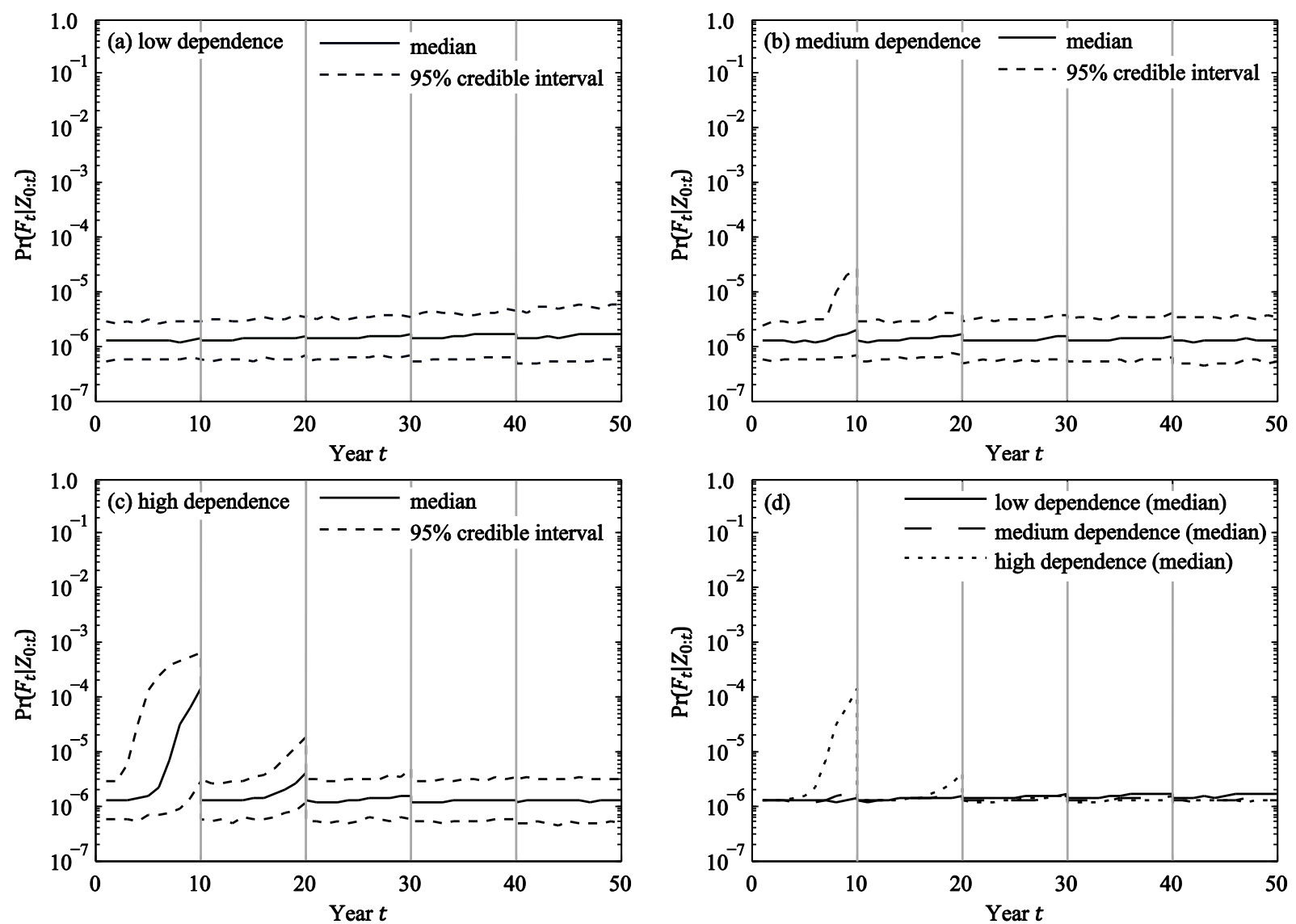

Figure 11. Median and 95\% credible interval of the posterior annual system failure probability $\operatorname{Pr}\left(F_{t} \mid Z_{0: t}\right)$ of the Daniels system as a function of different degrees of dependence among hotspot fatigue behavior. Hotspots $\{1$ to 10$\}$ are inspected at time $t=10$ years, hotspots $\{11$ to 20$\}$ are inspected at time $t=$ 20 years, hotspots $\{21$ to 30$\}$ are inspected at time $t=30$ years and hotspots $\{31$ to 40$\}$ are inspected at time 40 years. Each inspection results in a no-detection event. Computations are performed with subset simulation as summarized in Section 4 with conditional probabilities $p_{0}=0.1$ and $N=1000$ samples per subset level. (d) compares the respective medians of the posterior annual system failure probability.

\section{Discussion}

We propose a modeling and computational framework for analyzing the reliability of deteriorating structural systems and updating it with inspection and monitoring data. It enables an integral assessment of deterioration at the element level together with the structural system performance and structural condition information. The interdependences among the element deterioration states are included. Only few previous works have addressed such an integral system analysis (e.g. Lee and Song 2014; Schneider et al. 2015; Luque and Straub 2016). In contrast to these approaches, the main advantage of the proposed framework is the fact that it can be implemented easily through the use of subset simulation. It is computationally robust since it provides reasonably accurate solutions without a need for tailoring the algorithm to specific applications. It is also computationally efficient for many applications, as discussed further below. 
The results in the paper demonstrate the importance of considering dependence among element deterioration when evaluating the structural system reliability. For the considered redundant systems, the dependence leads to a decrease in the prior (unconditional) system reliability. This effect is more pronounced as the system redundancy increases (from the Zayas frame to the Daniels system). When including inspection results, dependence among element deterioration means that the state of non-inspected elements can be inferred from the inspection results. As long as inspections do not indicate serious problems, this additional learning leads to a reduction of uncertainty and hence to an increase in the overall system reliability. In the considered case studies, the posterior (conditional) reliability after the inspections is fairly similar for the different degrees of dependence. However, this is not expected to occur if inspections do indicate larger damage.

The framework can handle any type of information on the deterioration state of the structure, as long as a corresponding likelihood function is formulated. In particular, the framework can also include information from monitoring systems. For monitoring systems, which provide potentially large amount of data, it might be beneficial to pre-process the data. In such a pre-processing step (e.g. a system identification), the probability of the observed data given the deterioration states of the structure is determined. This probability is the likelihood function that is inputted into Equations (34) and (35). Such an approach is similar to a two-stage Bayesian analysis for system identification (see Au and Zhang 2015).

The use of subset simulation is computationally rather efficient, as demonstrated in the case studies. Here, no attempt was made to optimize the efficiency of subset simulation. The number of samples per subset level was chosen such that the results have a reasonable accuracy. Their accuracies can always be improved or reduced by increasing or decreasing the number of samples per subset level. It should be noted, however, that the number of required subsets increases with increasing amount of information, i.e. with decreasing $\operatorname{Pr}\left(Z_{e, 0: t}\right)$.

The proposed framework relies on the separation of the computation of the system deterioration state $\mathbf{D}_{t}$ and structural system reliability conditional on $\mathbf{D}_{t}=\mathbf{d}_{t}$. Here, two situations must be distinguished: Applications, in which the conditional probability $\operatorname{Pr}\left(F_{t} \mid \mathbf{D}_{t}=\mathbf{d}_{t}\right)$ can be precomputed, and those in which it cannot. The former occurs if the numbers of distinct states in $\mathbf{D}_{t}$ is limited. If the structural system reliability analysis is demanding, it might take some computation time for establishing a database with all values of $\operatorname{Pr}\left(F_{t} \mid \mathbf{D}_{t}=\mathbf{d}_{t}\right)$, but this is typically not critical, as this computation must be carried out only once and the database can be used for all subsequent reliability updating calculations. If the number of states in $\mathbf{D}_{t}$ is too large to enable precomputation, because there are too many elements or because continuous damage states are considered, $\operatorname{Pr}\left(F_{t} \mid \mathbf{D}_{t}=\mathbf{d}_{t}\right)$ must be computed on the fly. If such calculations are inexpensive (e.g. through a FORM analysis), the separation of the system deterioration state and structural system reliability is still computationally beneficial. In cases where pre-computation of $\operatorname{Pr}\left(F_{t} \mid \mathbf{D}_{t}=\mathbf{d}_{t}\right)$ is not an option, and in which it is expensive to compute it on the fly, there are two possible strategies: (a) One can investigate the possibility of developing a response surface for 
$\operatorname{Pr}\left(F_{t} \mid \mathbf{D}_{t}=\mathbf{d}_{t}\right)$. Since $\mathbf{D}_{t}$ is typically discrete, many of the classical response surface techniques used in structural reliability will not be suitable. This is an area of future research. (b) Alternatively,

840 the proposed framework can be modified to solve the system deterioration updating and the system 841 reliability jointly. In this case, however, the advantages of the de-coupling are lost.

842 Potentials for further developments are seen in integrating the presented method into the 843 framework of pre-posterior decision analysis to identify optimal inspection, monitoring and 844 maintenance strategies for engineering structures (e.g. Straub and Faber 2005; Thöns and Faber 845 2013; Straub 2014).

\section{Conclusions}

847 We propose a novel approach to modeling and analyzing the system reliability of deteriorating structural systems in conjunction with structural condition information, which considers stochastic interdependence among the deterioration states of the structural elements. The approach provides the means to consistently utilize inspection and monitoring information on the deterioration state of structures to update the system failure probability. Through the application of subset simulation, the approach can be implemented relatively easily and is considerably more efficient than crude Monte Carlo simulation.

\section{Acknowledgements}

We acknowledge insightful discussions with Iason Papaioannou and Wolfgang Betz on the implementation of the adaptive MCMC sampling algorithm. The work of Daniel Straub is supported by the German Science Foundation (DFG) through Grant STR 1140/3-1.

\section{References}

Altamura, A. and D. Straub (2014). "Reliability assessment of high cycle fatigue under variable amplitude loading: review and solutions." Engineering Fracture Mechanics 121: 40-66.

Au, S.-K. and J. L. Beck (2001). "Estimation of small failure probabilities in high dimensions by subset simulation." Probabilistic Engineering Mechanics 16(4): 263-277.

Au, S.-K., F. A. DiazDelaO and I. Yoshida (2015). "Bayesian updating and model class selection with Subset Simulation." eprint arXiv:1510.06989.

$\mathrm{Au}$, S.-K. and F.-L. Zhang (2015). "Fundamental two-stage formulation for Bayesian system identification, Part I: General theory." Mechanical Systems and Signal Processing.

Betz, W., I. Papaioannou and D. Straub (2014a). "Numerical methods for the discretization of random fields by means of the Karhunen-Loève expansion." Computer Methods in Applied Mechanics and Engineering 271: 109-129.

Betz, W., I. Papaioannou and D. Straub (2014b). Adaptive variant of the BUS approach to Bayesian updating. 9th International Conference on Structural Dynamics (EURODYN 2014). Porto, Portugal. 
Ciampoli, M. (1998). "Time dependent reliability of structural systems subject to deterioration." Computers \& Structures 67(1-3): 29-35.

Daniels, H. E. (1945). "The statistical theory of the strength of bundles of threads. I." Proceedings of the Royal Society of London: 405-435.

Ditlevsen, O. and P. Bjerager (1986). "Methods of structural systems reliability." Structural Safety 3(3): 195-229.

Ditlevsen, O. and H. O. Madsen (1996). Structural Reliability Methods, John Wiley \& Sons Ltd.

Enright, M. P. and D. M. Frangopol (1999). "Condition prediction of deteriorating concrete bridges using Bayesian updating." Journal of Structural Engineering 125(10): 1118-1125.

Estes, A. and D. Frangopol (1999). "Repair Optimization of Highway Bridges Using System Reliability Approach." Journal of Structural Engineering 125(7): 766-775.

Faber, M. H., S. Engelund, J. D. Sørensen and A. Bloch (2000). Simplified and Generic Risk Based Inspection Planning. 19th Conference on Offshore Mechanics and Arctic Engineering (OMAE), New Orleans, Louisiana, USA.

Faber, M. H., D. Straub and M. A. Maes (2006). "A computational framework for risk assessment of RC structures using indicators." Computer-Aided Civil and Infrastructure Engineering 21(3): 216-230.

Fricke, W. (2003). "Fatigue analysis of welded joints: state of development." Marine Structures 16(3): 185-200.

Gollwitzer, S. and R. Rackwitz (1990). "On the reliability of Daniels systems." Structural Safety 7(2-4): 229-243.

Gurney, T. R. (1978). An analysis of some recent fatigue crack propagation data for steels subjected to pulsating tension loading. Report 1978E, The Welding Institute TWI, UK

Hergenröder, M. (1992). Zur statistischen Instandhaltungsplanung für bestehende Betonbauwerke bei Karbonatisierung des Betons und möglicher Korrosion der Bewehrung PhD, Technische Universität München.

Hohenbichler, M. and R. Rackwitz (1981). "Non-Normal Dependent Vectors in Structural Safety." Journal of the Engineering Mechanics Division 107(6): 1227-1238.

Hohenbichler, M. and R. Rackwitz (1983). "First-order concepts in system reliability." Structural Safety 1(3): 177-188.

Lee, Y.-J. and J. Song (2014). "System Reliability Updating of Fatigue-Induced Sequential Failures." Journal of Structural Engineering 140(3).

Li, C. Q. (1995). "Computation of the failure probability of deteriorating structural systems." Computers \& Structures 56(6): 1073-1079.

Li, Q., C. Wang and B. R. Ellingwood (2015). "Time-dependent reliability of aging structures in the presence of non-stationary loads and degradation." Structural Safety 52, Part A: 132141 .

Li, Y., T. Vrouwenvelder, G. H. Wijnants and J. Walraven (2004). "Spatial variability of concrete deterioration and repair strategies." Structural Concrete 5(3): 121-129.

Lin, Y. K. and J. N. Yang (1985). "A stochastic theory of fatigue crack propagation." AIAA Journal 23(1): 117-124.

Liu, P.-L. and A. Der Kiureghian (1986). "Multivariate distribution models with prescribed marginals and covariances." Probabilistic Engineering Mechanics 1(2): 105-112.

Luque, J., R. Hamann and D. Straub (2016). "Spatial probabilistic modeling of corrosion in ships structures." ASCE-ASME Journal of Risk and Uncertainty in Engineering Systems, Part B: Mechanical Engineering: submitted.

Luque, J. and D. Straub (2016). "Reliability analysis and updating of deteriorating systems with dynamic Bayesian networks." Structural Safety 62: 34-46. 
Madsen, H. O. (1987). Model updating in reliability theory. 5th International Conference on Applications of Statistics and Probability in Civil Engineering (ICASP 5). Vancouver, Canada.

Madsen, H. O. (1997). Stochastic modeling of fatigue crack growth and inspection. Probabilistic Methods for Structural Design. C. Guedes Soares, Kluwer Accademic Publishers, Netherlands.

Madsen, H. O., S. Krenk and N. C. Lind (1986). Methods of Structural Safety, Prentice Hall.

Maes, M. A. and M. Dann (2007). "Hierarchical Bayes methods for systems with spatially varying condition states." Canadian Journal of Civil Engineering 34(10): 1289-1298.

Malioka, V. (2009). Condition Indicators for the Assessment of Local and Spatial Deterioration of Concrete Structures. PhD, ETH Zurich.

Maljaars, J. and A. C. W. M. Vrouwenvelder (2014). "Probabilistic fatigue life updating accounting for inspections of multiple critical locations." International Journal of Fatigue 68: 24-37.

Melchers, R. E. (1999a). "Corrosion uncertainty modelling for steel structures." Journal of Constructional Steel Research 52(1): 3-19.

Melchers, R. E. (1999b). Structural Reliability Analysis and Prediction, John Wiley and Sons Ltd.

Moan, T. (2005). "Reliability-based management of inspection, maintenance and repair of offshore structures." Structure and Infrastructure Engineering 1(1): 33-62.

Moan, T. and R. Song (2000). "Implications of Inspection Updating on System Fatigue Reliability of Offshore Structures." Journal of Offshore Mechanics and Arctic Engineering 122(3): 173-180.

Moan, T., O. T. Vardal, N. C. Hellevig and K. Skjoldli (2000). "Initial crack depth and PoD values inferred from in-service observations of cracks in North Sea jackets." Journal of Offshore Mechanics and Arctic Engineering 122: 157-162.

Mori, Y. and B. Ellingwood (1993). "Reliability-Based Service-Life Assessment of Aging Concrete Structures." Journal of Structural Engineering 119(5): 1600-1621.

Naess, A., B. J. Leira and O. Batsevych (2009). "System reliability analysis by enhanced Monte Carlo simulation." Structural Safety 31(5): 349-355.

Papaioannou, I., W. Betz, K. Zwirgelmaier and D. Straub (2015). "MCMC Algorithms for Subset Simulation." Probabilistic Engineering Mechanics 41: 89-103.

Paris, P. C. and F. A. Erdogan (1963). "A critical analysis of crack propagation laws." Journal of Basic Engineering 85: 528-534.

Qin, J. and M. H. Faber (2012). "Risk Management of Large RC Structures within a Spatial Information System." Computer-Aided Civil and Infrastructure Engineering 27: 385-405.

Schneider, R., J. Fischer, M. Bügler, M. Nowak, S. Thöns, A. Borrmann and D. Straub (2015). "Assessing and updating the reliability of concrete bridges subjected to spatial deterioration - principles and software implementation." Structural Concrete 16(3): 356-365.

Schneider, R., S. Thöns, W. Rücker and D. Straub (2013). Effect of different inspection strategies on the reliability of Daniels systems subjected to fatigue. 11th International Conference on Structural Safety \& Reliability (ICOSSAR 2013). New York, USA.

Schuëller, G. I. and H. J. Pradlwarter (2007). "Benchmark study on reliability estimation in higher dimensions of structural systems - An overview." Structural Safety 29(3): 167-182.

Skallerud, B. and J. Amdahl (2002). Nonlinear analysis of offshore structures. UK, Reasearch Studies Press Ltd.

Song, J. and W.-H. Kang (2009). "System reliability and sensitivity under statistical dependence by matrix-based system reliability method." Structural Safety 31(2): 148-156.

Stewart, M. and D. Val (1999). "Role of Load History in Reliability-Based Decision Analysis of Aging Bridges." Journal of Structural Engineering 125(7): 776-783. 
Stewart, M. G. and J. A. Mullard (2007). "Spatial time-dependent reliability analysis of corrosion damage and the timing of first repair for RC structures." Engineering Structures 29(7): 1457-1464.

Straub, D. (2004). Generic Approaches to Risk Based Inspection Planning for Steel Structures, ETH Zürich.

Straub, D. (2011a). "Reliability updating with equality information." Probabilistic Engineering Mechanics 26(2): 254-258.

Straub, D. (2011b). Reliability updating with inspection and monitoring data in deteriorating reinforced concrete slabs. 11th International Conference on Applications of Statistics and Probability in Civil Engineering. Zurich, Switzerland.

Straub, D. (2014). "Value of Information Analysis with Structural Reliability Methods." Structural Safety 49: 75-85.

Straub, D. and A. Der Kiureghian (2010). "Bayesian network enhanced with structural reliability methods: Methodology." Journal of Engineering Mechanics 136(10): 1248-1258.

Straub, D. and A. Der Kiureghian (2011). "Reliability acceptance criteria for deteriorating elements of structural systems." Journal of Structural Engineering 137(12): 1573-1582.

Straub, D. and M. H. Faber (2003). Modeling Dependency in Inspection Performance. 9th International Conference on Applications of Statistics and Probability in Civil Engineering. San Francisco, California.

Straub, D. and M. H. Faber (2005). "Risk based inspection planning for structural systems." Structural Safety 27: 335-355.

Straub, D. and M. H. Faber (2007). "Temporal Variability in Corrosion Modeling and Reliability Updating." Journal of Offshore Mechanics and Arctic Engineering 129(4): 265-272.

Straub, D. and I. Papaioannou (2015a). Bayesian analysis for learning and updating geotechnical parameters and models with measurements. Risk and reliability in geotechnical engineering. K.-K. Phoon and J. Ching, CRC Press.

Straub, D. and I. Papaioannou (2015b). "Bayesian updating with structural reliability methods." Journal of Engineering Mechanics 141(3).

Straub, D., I. Papaioannou and W. Betz (2016). "Bayesian analysis of rare events." Journal of Computational Physics: accepted.

Tang, W. H. (1973). "Probabilistic updating of flaw information." Journal of Testing and Evaluation 1(6): 459-467.

Thoft-Christensen, P. and Y. Murotsu (1986). Application of Structural Systems Reliability Theory, Springer-Verlag.

Thoft-Christensen, P. and J. D. Sørensen (1987). "Optimal strategy for inspection and repair of structural systems." Civil Engineering Systems 4: 94-100.

Thöns, S. and M. H. Faber (2013). Assessing the Value of Structural Health Monitoring. 11th International Conference on Structural Safety \& Reliability (ICOSSAR 2013). New York, USA.

Ultiguide (1999). Best practice guidelines for use of non-linear methods in documentation of ultimate limit states for jacket type offshore structures, Det Norske Veritas (DNV), Norway

USFOS (2014). Ultimate Strength of Framed Offshore Structures. Version 8-7, USFOS A/S, Norway

Vrouwenvelder, A. C. W. M. (2004). Spatial correlation aspects in deterioration models. 2nd International Conference on Lifetime-Oriented Design Concepts. Bochum, Germany.

Wen, Y. K. and H.-C. Chen (1987). "On fast integration for time variant structural reliability." Probabilistic Engineering Mechanics 2(3): 156-162. 
1018 Zayas, V. A., S. A. Mahin and E. P. Popov (1980). Cyclic inelastic behavior of steel offshore structures. UCB/EERC-80/27, University of California, Berkley, USA 